\title{
Hybrid coupling of a one-dimensional Energy-Transport Schrödinger system
}

\author{
Clément Jourdana ${ }^{1}$, Paola Pietra ${ }^{2}$ and Nicolas Vauchelet ${ }^{3}$ \\ ${ }^{1}$ Université Grenoble Alpes, Laboratoire Jean Kuntzmann, \\ F-38000 Grenoble, France. \\ ${ }^{2}$ Istituto di Matematica Applicata e Tecnologie Informatiche E.Magenes, CNR, \\ Via Ferrata 1, 27100 Pavia, Italy. \\ ${ }^{3}$ UPMC Univ Paris 06, UMR 7598, Laboratoire Jacques-Louis Lions, \\ CNRS, UMR 7598, Laboratoire Jacques-Louis Lions and \\ INRIA Paris-Rocquencourt, EPI MAMBA \\ F-75005, Paris, France
}

\begin{abstract}
We consider one dimensional coupled classical-quantum models for quantum semiconductor device simulations. The coupling occurs in the space variable : the domain of the device is divided into a region with strong quantum effects (quantum zone) and a region where quantum effects are negligible (classical zone). In the classical zone, transport in diffusive approximation is modeled through diffusive limits of the Boltzmann transport equation. It can lead to an Energy-Transport model, obtained using a Spherical Harmonic Expansion model as intermediate step. The quantum transport is described by the Schrödinger equation. The aim of this work is to focus on the derivation of boundary conditions at the interface between the classical and quantum regions. Numerical simulations are provided for a resonant tunneling diode with the Energy-Transport model.
\end{abstract}

Keywords. Schrödinger equation, Boltzmann equation, energy-transport system, spherical harmonic expansion system, semiconductors, interface conditions, mixed finite elements.

AMS subject classifications. 65M60, 65Z05, 82D37, 82D80, 35J10, 76P05.

\section{Introduction}

Due to constant downscaling at nanometer scale of electronic components, quantum effects in the transport of charged carriers cannot be negligible. Among the observed quantum phenomena we can cite confinement, tunneling effect or interferences. To take into account such phenomena, an accurate description of the quantum transport of charged carriers should be considered, so that electrons are no more described as point particles but rather as waves. The Schrödinger equation is usually used for the modeling. However, for 
instance for resonant tunneling diodes, quantum effects are well localized in a small region of the device whereas classical transport is relevant to describe the transport of charged carriers in the rest of the device. Moreover, from a numerical point of view, numerical simulations of the Schrödinger equation are costly and it is then interesting to restrict its use to the region where the quantum effects occur.

We consider in this work those devices for which quantum effects are localized in a well defined region, which is referred to as the quantum zone. The rest of the device domain is called the classical zone. In the quantum zone, the Schrödinger equation is used to describe electrons transport. In the classical zone, the transport is assumed to be purely classical and mainly driven by collisions. Then, the coupling between the models is obtained through boundary conditions at the interface between these two regions. In his seminal work [4], N. Ben Abdallah proposes a coupled kinetic-quantum model where the Schrödinger equation was used to compute the density in the quantum zone whereas, in the classical regions, a Boltzmann equation is used to describe the collisional transport of electrons in the rest of the domain. At the classical-quantum interface, boundary conditions for the Boltzmann equation depending on the quantum reflection and transmission coefficients have been defined. A numerical discretization of this approach has been later proposed in [12]. In the aim to diminish computational cost, the diffusive limit of the Botzmann equation leading to the drift-diffusion (DD) model has been considered in [15]. In their work, the interface boundary conditions have been obtained by passing to the limit in the reflection-transmission conditions obtained in [4] and by considering boundary layer corrections leading to the resolution to a Milne problem. Another approach has been proposed in [3] by imposing the continuity of the current at the interfaces.

We propose in this paper to extend the aforementioned study to the case where an energy-transport (ET) model is used in the classical region of a semiconductor. Such model may be derived by a diffusive limit departing from the Boltzmann transport equation (BTE), through a Spherical Harmonic Expansion (SHE) system as intermediate step, as proposed in [6]. The BTE governs the dynamics of the distribution function of the electrons denoted $f(t, x, k)$ where $t>0$ is the time variable, $x$ is the space variable and $k \in B$ is the momentum variable belonging to the Brillouin zone. In semiconductor devices, transport of electrons can be strongly affected by collisions. Among them, we can distinguish elastic collisions with impurity of the lattice, collisions between electrons and collisions with phonons. When elastic collisions are the dominant scattering mechanism, the BTE can be approximated by the SHE model (see e.g. [39]). This model has been first introduced in [36] and governs the dynamics of the distribution function $F(t, x, \mathrm{E})$ as a function of the energy E. Assuming then a dominant electron-electron scattering, the SHE model relaxes to the so-called energy-transport system. The macroscopic quantities considered in this system are the density and the temperature. This system has been introduced in [36, 37] and can be also derived directly from the Boltzmann equation under the assumption of dominant elastic and electron-electron collisions (see e.g. [7]). However, for numerical purpose, it appears to be interesting to consider the system obtained from the SHE model [16]. Finally, under the assumption of large electron-phonon collision mechanism, the ET system converges to the drift-diffusion system for the density of electron where the temperature is constant, equal to the lattice temperature. 
The aim of this paper is to discuss the interface boundary conditions that can be used to tackle a one dimensional hybrid coupling in the space variable between the ET model and the Schrödinger equation. As intermediate step, we also consider the coupling between the SHE model and the Schrödinger equation. Imposing the continuity of the current at interfaces, we recover, at the diffusive limit, the interface conditions of [15, 3] for the driftdiffusion/Schrödinger coupling. However, compared to the DD model, the ET model give a more precise description of kinetic effects which can appear in the domain. On another hand, passing through the SHE model, we are able to derive interface conditions between the classical and the quantum regions directly thanks to an approximation of the boundary conditions for the kinetic model. Throughout this paper, we will attach importance to make a link between these two approaches. In particular, numerical results will be obtained for the ET-Schrödinger coupling thanks to the two different interface condition approaches.

Several authors have also considered interface problems between macroscopic models to describe the classical transport. The case of drift-diffusion systems has been introduced in [40] and has been extended to other macroscopic models in [20]. In [19], the authors consider the case of space-dependent band diagrams and abrupt heterojunction. Fluid models taking into account localized kinetic effects are obtained and simulated in [17. Finally, in the framework of strongly confined nanostructures, a hybrid approach [27] has been recently proposed to spatially couple a multiband Schrödinger system [8] with a nanowire drift-diffusion model [28], preserving the continuity of the current.

We conclude this introduction by referring to other quantum/classical coupling. In devices where particles are strongly confined in one direction, whereas the other directions can be considered as classical, models where the coupling occurs in the momentum variable are proposed in [10, 5, 11, 34]. Such approach can be obtained thanks to a semiclassical limit of the Schrödinger equation with a partially confining potential [9]. Quantum energy-transport and quantum drift-diffusion models have been derived in [18] using the strategy of quantum moments, as well as in [30. These models involve a quantum chemical potential that depends on the density in a non-local way.

Throughout the paper, we restrict ourselves to the one-dimensional case. We denote by $[0, L]$ the domain where the device lies and by $x$ the transport variable. The quantum zone is $Q=\left[x_{1}, x_{2}\right]$ and the classical zone is then $C=\left[0, x_{1}\right] \cup\left[x_{2}, L\right]$. The quantum transport in $Q$ is then described by a Schrödinger equation for the scattering states. The classical transport is modeled by a hierarchy of macroscopic models derived from the Boltzmann transport equation. The coupling between these models occurs then for $x=x_{1}$ and $x=x_{2}$.

The outline of the paper is the following. In Section 2, we describe the quantum region and recall the expressions of useful macroscopic quantities. Section 3 is devoted to the classical region. The one-dimensional Boltzmann transport equation is recalled; its diffusive limit towards the SHE model, then the ET and the DD systems are proposed. In Section 4, we derive the interface conditions at the boundary $x=x_{1}$ and $x=x_{2}$. We describe the two approaches that we propose : the one based on the computation of boundary layers leading to a Milne problem and the one based on the continuity of the current. Then, we consider in Section 5 the numerical discretization of this problem 
and present the algorithm for numerical simulations of the hybrid model presented in this work. Finally numerical results are provided in Section 6 for the simulation of a one-dimensional resonant tunneling diode with the ET model.

\section{Quantum region}

In the quantum region $Q=\left[x_{1}, x_{2}\right]$, we consider the scattering states of the Schrödinger equation in the potential energy $V$ (possibly depending on $t$ ) :

$$
\begin{array}{ll}
-\frac{1}{2 m} \partial_{x x} \psi_{k}+V(x) \psi_{k}=\epsilon\left(x_{1}, k\right) \psi_{k}, & \text { for } k>0, \\
-\frac{1}{2 m} \partial_{x x} \psi_{k}+V(x) \psi_{k}=\epsilon\left(x_{2}, k\right) \psi_{k}, & \text { for } k<0 .
\end{array}
$$

where $m$ is the effective mass and $\epsilon$ is the total particle energy of an electron in the lattice. It is the sum of the kinetic energy $\varepsilon$ and of the potential energy :

$$
\epsilon(x, k)=\varepsilon(k)+V(x)=\frac{k^{2}}{2 m}+V(x) .
$$

This system is complemented with the Transparent Boundary Conditions TBCs [4] :

$$
\begin{array}{ll}
\partial_{x} \psi_{k}\left(x_{1}\right)=2 i k-i k \psi_{k}\left(x_{1}\right), & \partial_{x} \psi_{k}\left(x_{2}\right)=i k_{+}(k) \psi_{k}\left(x_{2}\right), \quad \text { for } k>0, \\
\partial_{x} \psi_{k}\left(x_{1}\right)=-i k_{-}(k) \psi_{k}\left(x_{1}\right), & \partial_{x} \psi_{k}\left(x_{2}\right)=2 i k-i k \psi_{k}\left(x_{2}\right), \quad \text { for } k<0 .
\end{array}
$$

This system is equivalent to the resolution of the Schrödinger equations (2.1)-(2.2) on the real line $\mathbb{R}$ with a potential $\widetilde{V}$ defined as the extension by continuity of the potential $V$ into constant functions outside $Q$. In fact, in this case the scattering states, which are defined as solutions of (2.1)-2.2), satisfy for $k>0$ :

$$
\begin{aligned}
& \psi_{k}(x)=e^{i k\left(x-x_{1}\right)}+r(k) e^{-i k\left(x-x_{1}\right)}, \text { for } x<x_{1}, \\
& \psi_{k}(x)=t(k) e^{i k_{+}(k)\left(x-x_{2}\right)}, \text { for } x>x_{2} .
\end{aligned}
$$

And for $k<0$ :

$$
\begin{aligned}
& \psi_{k}(x)=e^{i k\left(x-x_{2}\right)}+r(k) e^{-i k\left(x-x_{2}\right)}, \text { for } x>x_{2}, \\
& \psi_{k}(x)=t(k) e^{-i k_{-}(k)\left(x-x_{1}\right)}, \text { for } x<x_{1} .
\end{aligned}
$$

From now on, $V_{1}$ (resp. $\left.V_{2}\right)$ stands for $V\left(x_{1}\right)$ (resp. $V\left(x_{2}\right)$ ). In all the presentation, we will assume that $V_{1} \geq V_{2}$. In the above equations we have defined :

$$
k_{+}(k)=\sqrt{k^{2}+2 m\left(V_{1}-V_{2}\right)}, \quad k_{-}(k)=\sqrt{k^{2}-2 m\left(V_{1}-V_{2}\right)},
$$

where $\sqrt{\alpha}$ is the complex square root of the real number $\alpha$ having a positive real part (if $\alpha>0$ ) or a positive imaginary part (if $\alpha<0$ ). We define the reflection and transmission amplitudes by

$$
\begin{aligned}
r(k) & =\frac{1}{2}\left(\psi_{k}+\frac{i}{k} \partial_{x} \psi_{k}\right)\left(x_{1}\right), & t(k)=\psi_{k}\left(x_{2}\right), & \text { for } k>0, \\
r(k) & =\frac{1}{2}\left(\psi_{k}+\frac{i}{k} \partial_{x} \psi_{k}\right)\left(x_{2}\right), & t(k)=\psi_{k}\left(x_{1}\right), & \text { for } k<0 .
\end{aligned}
$$


Then, the reflection and transmission coefficients $\mathcal{R}(k)$ and $\mathcal{T}(k)$ are defined by

$$
\begin{gathered}
\mathcal{R}(k)=|r(k)|^{2}, \quad k \in \mathbb{R} ; \quad \mathcal{T}(k)=k_{+}(k) \frac{|t(k)|^{2}}{k}, \quad \text { for } k>0, \\
\mathcal{T}(k)=-\mathfrak{R} e\left(k_{-}(k)\right) \frac{|t(k)|^{2}}{k}, \quad \text { for } k<0 .
\end{gathered}
$$

They satisfy the reciprocity relations:

$$
\begin{array}{cl}
\mathcal{T}(k)+\mathcal{R}(k)=1, & \text { for } k \in \mathbb{R}, \\
\mathcal{R}(k)=1, & \text { for }-k_{+}(0) \leq k \leq 0, \\
\mathcal{T}(k)=\mathcal{T}\left(-k_{+}(k)\right), & \text { for } k>0, \\
\mathcal{T}(k)=\mathcal{T}\left(k_{-}(k)\right), & \text { for } k<-k_{+}(0) .
\end{array}
$$

We assume that the distribution function $f$ in the classical region $C$ is known. Its values $f\left(x_{1}, k\right)$ for $k>0$ and $f\left(x_{2}, k\right)$ for $k<0$ correspond to particles entering the quantum region $Q$ and are used as alimentation functions to construct the quantum electron density $N_{Q}$ defined by

$$
N_{Q}(x)=\int_{k>0} f\left(x_{1}, k\right)\left|\psi_{k}(x)\right|^{2} d k+\int_{k<0} f\left(x_{2}, k\right)\left|\psi_{k}(x)\right|^{2} d k, \quad x \in Q .
$$

The particle current in the $Q$ region is

$$
J_{Q}=\int_{k>0} f\left(x_{1}, k\right) \mathcal{I} m\left(\frac{1}{m} \partial_{x} \psi_{k}(x) \overline{\psi_{k}(x)}\right) d k+\int_{k<0} f\left(x_{2}, k\right) \mathcal{I} m\left(\frac{1}{m} \partial_{x} \psi_{k}(x) \overline{\psi_{k}(x)}\right) d k
$$

We can reformulate this current in term of the transmission coefficients :

Lemma 2.1 Assume, as above, that $V_{1} \geq V_{2}$. If $\left(\psi_{k}\right)_{k}$ are solutions to the Schrödinger equations (2.1)-(2.2) with transparent boundary conditions (2.4)-(2.5). Then, the particle current $J_{Q}$ defined in 2.12 satisfies :

$$
J_{Q}=\int_{0}^{+\infty} \frac{1}{m}\left(f\left(x_{1}, k\right)-f\left(x_{2},-k_{+}(k)\right)\right) k \mathcal{T}(k) d k .
$$

Moreover, introducing the notation

$$
k(x, E)=\sqrt{2 m(E-V(x))}, \quad \text { for } E>V(x),
$$

we have $J_{Q}=\int_{V_{1}}^{+\infty} \mathcal{J}_{Q}(E) d E$, where

$$
\mathcal{J}_{Q}(E)=\left(f\left(x_{1}, k\left(x_{1}, E\right)\right)-f\left(x_{2},-k\left(x_{2}, E\right)\right)\right) \mathcal{T}\left(k\left(x_{1}, E\right)\right) .
$$

Proof. Formula (2.13) is well-known (see e.g. [4]), but for completeness we recall the derivation here. It is quite standard and straightforward from the Schrödinger equation 
(2.1) - 2.2 that the quantity $\operatorname{Im}\left(\partial_{x} \psi_{k}(x) \overline{\psi_{k}(x)}\right)$ does not depend on $x$. Therefore, for $k>0$, we have from the boundary conditions $(2.4)$

$$
\mathcal{I} m\left(\partial_{x} \psi_{k}(x) \overline{\psi_{k}(x)}\right)=\mathcal{I} m\left(\partial_{x} \psi_{k}\left(x_{2}\right) \overline{\psi_{k}\left(x_{2}\right)}\right)=\mathcal{I} m\left(i k_{+}(k)\left|\psi_{k}\left(x_{2}\right)\right|^{2}\right)=k \mathcal{T}(k),
$$

where we use the definition of transmission amplitude $\mathcal{T}(k) 2.6$ in the last identity. For $k<0$, we have from 2.5

$$
\mathcal{I} m\left(\partial_{x} \psi_{k}(x) \overline{\psi_{k}(x)}\right)= \begin{cases}-k_{-}(k)\left|\psi_{k}\left(x_{1}\right)\right|^{2}, & \text { if } k<-k_{+}(0), \\ 0, & \text { if }-k_{+}(0)<k<0\end{cases}
$$

Therefore, using the definition (2.6), we have

$$
\operatorname{Im}\left(\partial_{x} \psi_{k}(x) \overline{\psi_{k}(x)}\right)= \begin{cases}k \mathcal{T}(k), & \text { if } k<-k_{+}(0) \\ 0, & \text { if }-k_{+}(0)<k<0\end{cases}
$$

Then, we can rewrite

$$
\int_{k<0} f\left(x_{2}, k\right) \mathcal{I} m\left(\partial_{x} \psi_{k}(x) \overline{\psi_{k}(x)}\right) d k=\int_{-\infty}^{-k_{+}(0)} f\left(x_{2}, k\right) k \mathcal{T}(k) d k
$$

Using the change of variables $k^{\prime}=k_{-}(k)$, which implies $k^{\prime} d k^{\prime}=k d k$, we have $k \mathcal{T}(k) d k=$ $k^{\prime} \mathcal{T}\left(-k_{+}\left(k^{\prime}\right)\right) d k^{\prime}$. From the reciprocity relation $(2.9)$, we deduce

$$
\int_{k<0} f\left(x_{2}, k\right) \mathcal{I} m\left(\partial_{x} \psi_{k}(x) \overline{\psi_{k}(x)}\right) d k=-\int_{0}^{+\infty} f\left(x_{2},-k_{+}\left(k^{\prime}\right)\right) k^{\prime} \mathcal{T}\left(k^{\prime}\right) d k^{\prime} .
$$

This proves formula 2.13 .

Then, we use the definition (2.14). If $\mathrm{E}>V_{1}$, we set $k_{1}(\mathrm{E})=k\left(x_{1}, \mathrm{E}\right)$ and $k_{2}(\mathrm{E})=$ $k\left(x_{2}, \mathrm{E}\right)$ (we recall that we assume $\left.V_{1} \geq V_{2}\right)$. We have $k_{2}(\mathrm{E})=k_{+}\left(k_{1}(\mathrm{E})\right)$, so that $k_{2}(\mathrm{E})>$ $k_{+}(0)$ for $\mathrm{E}>V_{1}$. We define, for $\mathrm{E}>V_{1}$,

$$
\mathcal{J}_{Q}(\mathrm{E})=f\left(x_{1}, k_{1}(\mathrm{E})\right) \frac{\mathcal{I} m\left(\partial_{x} \psi_{k_{1}}(x) \overline{\psi_{k_{1}}(x)}\right)}{\left|k_{1}(\mathrm{E})\right|}+f\left(x_{2},-k_{2}(\mathrm{E})\right) \frac{\mathcal{I} m\left(\partial_{x} \psi_{-k_{2}}(x) \overline{\psi_{-k_{2}}(x)}\right)}{\left|k_{2}(\mathrm{E})\right|}
$$

so that, we have clearly

$$
\int_{V_{1}}^{+\infty} \mathcal{J}_{Q}(\mathrm{E}) d \mathrm{E}=J_{Q},
$$

where $J_{Q}$ is defined in 2.12. Then, from 2.16 and 2.17), we have

$$
\mathcal{J}_{Q}(\mathrm{E})=\operatorname{sgn}\left(k_{1}(\mathrm{E})\right) f\left(x_{1}, k_{1}(\mathrm{E})\right) \mathcal{T}\left(k_{1}(\mathrm{E})\right)-\operatorname{sgn}\left(k_{2}(\mathrm{E})\right) f\left(x_{2},-k_{2}(\mathrm{E})\right) \mathcal{T}\left(-k_{2}(\mathrm{E})\right),
$$

where sgn denotes the sign function. Since $k_{2}(\mathrm{E})=k_{+}\left(k_{1}(\mathrm{E})\right)$, we deduce 2.15) from the reciprocity relation 2.9 . 


\section{Classical region}

\subsection{Description of the kinetic regime}

We consider the one dimensional Boltzmann transport equation for semiconductors [6] in scaled form

$$
\partial_{t} f^{\alpha}+\frac{1}{\alpha} \frac{k}{m} \partial_{x} f^{\alpha}-\frac{1}{\alpha} \partial_{x} V \partial_{k} f^{\alpha}=\frac{1}{\alpha^{2}}\left(Q_{i m p}\left(f^{\alpha}\right)+Q_{p h}^{\alpha}\left(f^{\alpha}\right)\right)+\frac{1}{\beta} Q_{e}\left(f^{\alpha}\right),
$$

where $f^{\alpha}=f^{\alpha}(t, x, k)$. In this equation $x \in C=\left[0, x_{1}\right] \cup\left[x_{2}, L\right]$ and $k \in B$ where $B$ is the Brillouin zone. $\alpha$ and $\beta$ are dimensionless parameters that satisfy $\alpha<<\beta<<1$. $Q_{\text {imp }}$ is an elastic collision operator, accounting for collisions with the impurities of the lattice. It writes

$$
Q_{i m p}(f)=\int_{B} \phi_{i m p}\left(k, k^{\prime}\right) \delta\left(\varepsilon^{\prime}-\varepsilon\right)\left(f^{\prime}-f\right) d k^{\prime},
$$

where $\varepsilon$ and $\varepsilon^{\prime}$ (resp. $f$ and $f^{\prime}$ ) stand for $\varepsilon(k)$ and $\varepsilon\left(k^{\prime}\right)$ (resp. $f(k)$ and $f\left(k^{\prime}\right)$ ). $\varepsilon(k)$ is the kinetic energy defined in (2.3) and $\delta$ is the Dirac measure. The electron-electron collision operator $Q_{e}$ is given by

$$
\begin{gathered}
Q_{e}(f)=\int_{B^{3}} \phi_{e}\left(k, k_{1}, k^{\prime}, k_{1}^{\prime}\right) \delta\left(\varepsilon\left(k^{\prime}\right)+\varepsilon\left(k_{1}^{\prime}\right)-\varepsilon(k)-\varepsilon\left(k_{1}\right)\right) \times \\
\left(f\left(k^{\prime}\right) f\left(k_{1}^{\prime}\right)(1-\eta f(k))\left(1-\eta f\left(k_{1}\right)\right)-f(k) f\left(k_{1}\right)\left(1-\eta f\left(k^{\prime}\right)\right)\left(1-\eta f\left(k_{1}^{\prime}\right)\right)\right) d k^{\prime} d k_{1} d k_{1}^{\prime} .
\end{gathered}
$$

$\eta \geq 0$ is a dimensionless distribution function scaling factor and the terms $0 \leq 1-\eta f \leq 1$ express the Pauli exclusion principle. Collisions with phonons (acoustical and optical) are described thanks to the operator $Q_{p h}^{\alpha}$ :

$$
\begin{aligned}
Q_{p h}^{\alpha}(f) & =\int_{B} \phi_{p h}\left(k, k^{\prime}\right)\left[\left(\left(N_{p h}^{\alpha}+1\right) \delta\left(\varepsilon-\varepsilon^{\prime}+\alpha^{2} \varepsilon_{p h}\right)+N_{p h}^{\alpha} \delta\left(\varepsilon-\varepsilon^{\prime}-\alpha^{2} \varepsilon_{p h}\right)\right) f^{\prime}(1-\eta f)\right. \\
& \left.-\left(\left(N_{p h}^{\alpha}+1\right) \delta\left(\varepsilon^{\prime}-\varepsilon+\alpha^{2} \varepsilon_{p h}\right)+N_{p h}^{\alpha} \delta\left(\varepsilon^{\prime}-\varepsilon-\alpha^{2} \varepsilon_{p h}\right)\right) f\left(1-\eta f^{\prime}\right)\right] d k^{\prime}
\end{aligned}
$$

$\varepsilon_{p h}$ is the phonon energy and $N_{p h}^{\alpha}=\left(e^{\alpha^{2} \varepsilon_{p h}}-1\right)^{-1}$ is the phonon occupation number. We can expand the latter collision operator $Q_{p h}^{\alpha}$ in term of $\alpha^{2}: Q_{p h}^{\alpha}(f)=Q_{p h}^{0}(f)+\alpha^{2} Q_{1}^{\alpha}(f)$. Then, we define the elastic collision operator $Q_{0}$ by

$$
Q_{0}(f)=\int_{B} \phi_{0}\left(k, k^{\prime}\right) \delta\left(\varepsilon^{\prime}-\varepsilon\right)\left(f^{\prime}-f\right) d k^{\prime},
$$

where $\phi_{0}=\phi_{i m p}+\left(2 N_{p h}+1\right) \phi_{p h}$ with $N_{p h}$ the 0-th order term of $N_{p h}^{\alpha}$.

In all these definitions, the scattering coefficients $\phi_{i m p}, \phi_{e}$ and $\phi_{p h}$ are nonnegative quantities. Also, in the sequel, we shall assume the following micro-reversibility properties

$$
\begin{gathered}
\phi_{0}\left(k, k^{\prime}\right)=\phi_{0}\left(k^{\prime}, k\right) \text { for all } k, k^{\prime} . \\
\phi_{e}\left(k, k_{1}, k^{\prime}, k_{1}^{\prime}\right)=\phi_{e}\left(k_{1}, k, k^{\prime}, k_{1}^{\prime}\right)=\phi_{e}\left(k^{\prime}, k_{1}^{\prime}, k, k_{1}\right), \text { for all } k, k_{1}, k^{\prime}, k_{1}^{\prime} .
\end{gathered}
$$


Notice that (3.5) implies that $\phi_{0}(k,-k)$ is only dependent on the modulus of $k$ (and on $x$ ).

Finally the Boltzmann transport equation for semiconductors writes

$$
\partial_{t} f^{\alpha}+\frac{1}{\alpha} \frac{k}{m} \partial_{x} f^{\alpha}-\frac{1}{\alpha} \partial_{x} V \partial_{k} f^{\alpha}=\frac{1}{\alpha^{2}} Q_{0}\left(f^{\alpha}\right)+\frac{1}{\beta} Q_{e}\left(f^{\alpha}\right)+Q_{1}^{\alpha}\left(f^{\alpha}\right), \quad t>0, x \in C, k \in B .
$$

The system should be complemented with boundary condition for $x \in\left\{0, x_{1}, x_{2}, L\right\}$. At $x=0$ and $x=L$, the inflow particles are assumed to be prescribed :

$$
f^{\alpha}(0, k)=f^{i n}(k), \quad \text { for } k>0 ; \quad f^{\alpha}(L, k)=f^{i n}(k), \quad \text { for } k<0 .
$$

At $x=x_{1}$ and $x=x_{2}$ the interface with the quantum region should be taken into account. In particular particles can be transmitted or reflected through this region. It is proved in [4] that these conditions write

$$
\left\{\begin{array}{l}
f^{\alpha}\left(x_{1}, k\right)=\mathcal{R}(-k) f^{\alpha}\left(x_{1},-k\right)+\mathcal{T}\left(-k_{+}(k)\right) f^{\alpha}\left(x_{2},-k_{+}(k)\right), \quad \text { for } k<0 \\
f^{\alpha}\left(x_{2}, k\right)=\mathcal{R}(-k) f^{\alpha}\left(x_{2},-k\right)+\mathcal{T}\left(k_{-}(k)\right) f^{\alpha}\left(x_{1}, k_{-}(k)\right), \quad \text { for } k>k_{+}(0), \\
f^{\alpha}\left(x_{2}, k\right)=f^{\alpha}\left(x_{2},-k\right), \quad \text { for } 0<k<k_{+}(0),
\end{array}\right.
$$

providing current conservation on the entire domain. For the sake of simplicity of the notations, we will synthetise the interface conditions (3.8) as

$$
\mathcal{B}\left(f_{1}^{\alpha}, f_{2}^{\alpha}\right)=0
$$

where $f_{i}^{\alpha}(k):=f^{\alpha}\left(x_{i}, k\right), i=1,2$.

Although the derivation of the interface conditions holds in a general framework, for the sake of simplicity, we will only consider the parabolic band approximation. We mention however that such approximation proves to be not accurate for high fields (see e.g. [33, 13]). In this setting and in one dimension, we have $B=\mathbb{R}$, hence the kinetic model simplifies. In fact, the set of constant energy $\{\epsilon(x, k)=\mathrm{E}\}$ is given by $\{k(x, \mathrm{E}),-k(x, \mathrm{E})\}$ with definition (2.14). For a function $f$, it will be useful to define the even part of this function by

$$
F(\mathrm{E})=\frac{1}{2}(f(\sqrt{2 m(\mathrm{E}-V)})+f(-\sqrt{2 m(\mathrm{E}-V)})), \quad \forall \mathrm{E} \geq V
$$

In other words, we have

$$
F\left(\frac{k^{2}}{2 m}+V\right)=\frac{1}{2}(f(k)+f(-k)), \quad \forall k \in \mathbb{R} .
$$

Also, we can use the fact that, for a function $h$,

$$
\int_{B} h\left(k^{\prime}\right) \delta\left(\varepsilon-\varepsilon^{\prime}\right) d k^{\prime}=\frac{m}{|k|}(h(k)+h(-k)) .
$$


Consequently, the elastic collision operator $Q_{0}(3.4)$ simplifies as

$$
Q_{0}(f)(k)=\phi_{0}(k,-k)(f(-k)-f(k)) \frac{m}{|k|} .
$$

Similar transformations can be done for the other collision terms. The collision operator $Q_{0}$ has standard properties (see e.g. [6]). In particular, under the micro-reversibility assumption (3.5), we have

$$
\text { Ker } Q_{0}=\left\{f \text { even, i.e. } f(k)=F\left(\frac{k^{2}}{2 m}+V\right)\right\} \text { and } \mathrm{R}\left(Q_{0}\right)=\left(\operatorname{Ker} Q_{0}\right)^{\perp}=\{f \text { odd }\} \text {. }
$$

\subsection{Derivation of the one dimensional SHE model}

The dimensionless parameter $\alpha>0$ compares the inelastic and electron-electron interactions to the elastic collisions. When $\alpha \rightarrow 0$ the elastic interactions dominate and we can derive the so-called SHE model. To do that, let us consider the following Hilbert expansion

$$
f^{\alpha}=f^{0}+\alpha f^{1}+\alpha^{2} f^{2}+\ldots
$$

Injecting this expression in (3.7) and identifying the terms with equal powers of $\alpha$, we get

$$
\begin{gathered}
Q_{0}\left(f^{0}\right)=0 \\
Q_{0}\left(f^{1}\right)=\frac{k}{m} \partial_{x} f^{0}-\partial_{x} V \partial_{k} f^{0} \\
\partial_{t} f^{0}+\frac{k}{m} \partial_{x} f^{1}-\partial_{x} V \partial_{k} f^{1}=Q_{0}\left(f^{2}\right)+\frac{1}{\beta} Q_{e}\left(f^{0}\right)+Q_{1}^{\alpha}\left(f^{0}\right) .
\end{gathered}
$$

From (3.13) and using the property $(3.12)$, we deduce that $f^{0}$ is a function of the energy :

$$
f^{0}(x, k)=F^{0}(x, \epsilon(x, k)) .
$$

With this expression, we have by the chain rule

$$
\begin{aligned}
\frac{k}{m} \partial_{x} f^{0}-\partial_{x} V \partial_{k} f^{0} & =\frac{k}{m} \partial_{x}\left(F^{0}(x, \epsilon(x, k))\right)-\partial_{x} V \partial_{k} \epsilon(x, k) \partial_{\epsilon} F^{0}(x, \epsilon(x, k)) \\
& =\frac{k}{m} \partial_{x} F^{0}(x, \epsilon(x, k))+\left(\frac{k}{m} \partial_{x} \epsilon(x, k)-\partial_{x} V \partial_{k} \epsilon(x, k)\right) \partial_{\epsilon} F^{0}(x, \epsilon(x, k)) .
\end{aligned}
$$

The last term vanishes due to the definition of $\epsilon(x, k)$ in (2.3). Then, (3.14) rewrites

$$
Q_{0}\left(f^{1}\right)=\frac{k}{m} \partial_{x} F^{0}(x, \epsilon(x, k)) .
$$

For every $h \in \mathrm{R}\left(Q_{0}\right)$, the equation $Q_{0}(f)=h$ has a unique solution $f \in \mathrm{R}\left(Q_{0}\right)$. So, we introduce the function $\lambda \in \mathrm{R}\left(Q_{0}\right)$ such that $Q_{0}(\lambda)=-\frac{k}{m}$. We have

$$
\lambda(x, k)=\frac{k|k|}{2 m^{2} \phi_{0}(x, k,-k)}
$$


and we deduce that the solution of (3.17) can be written as

$$
f^{1}(x, k)=-\lambda(x, k) \partial_{x} F^{0}(x, \epsilon(x, k))+F^{1}(x, \epsilon(x, k)),
$$

for any real-valued function $F^{1}$. Next, (3.15) leads to

$$
\partial_{t} F^{0}+\partial_{t} V \partial_{\mathrm{E}} F^{0}+\frac{k}{m} \partial_{x} f^{1}-\partial_{x} V \partial_{k} f^{1}-\frac{1}{\beta} Q_{e}\left(F^{0}\right)-Q_{1}^{\alpha}\left(F^{0}\right)=Q_{0}\left(f^{2}\right) .
$$

This equation admits a solution $f^{2}$ provided the left hand side is an odd function, that is

$$
\begin{gathered}
\frac{2 m}{|k|}\left(\partial_{t} F^{0}+\partial_{t} V \partial_{\mathrm{E}} F^{0}\right)-\partial_{x} J_{S H E}=\frac{1}{\beta} S_{e}\left(F^{0}\right)+S_{1}^{\alpha}\left(F^{0}\right), \\
J_{S H E}(x, \epsilon(x, k))=D(x, k) \partial_{x} F^{0}(x, \epsilon(x, k)),
\end{gathered}
$$

where the diffusion coefficient is given by

$$
D(x, k)=2 \lambda(x,|k|),
$$

and the scattering operators are

$$
\begin{gathered}
S_{e}\left(F^{0}\right)(x, \mathrm{E})=S_{e}\left(F^{0}\right)(x, k,-k)=\frac{2 m}{|k|}\left(Q_{e}\left(F^{0}\right)(x, k)+Q_{e}\left(F^{0}\right)(x,-k)\right), \\
S_{1}^{\alpha}\left(F^{0}\right)(x, \mathrm{E})=S_{1}^{\alpha}\left(F^{0}\right)(x, k,-k)=\frac{2 m}{|k|}\left(Q_{1}^{\alpha}\left(F^{0}\right)(x, k)+Q_{1}^{\alpha}\left(F^{0}\right)(x,-k)\right) .
\end{gathered}
$$

Finally, the solution of $(3.15)$ is given by

$$
f^{2}(x, k)=-\frac{|k|}{2 m \phi_{0}(x, k,-k)}\left(\frac{k}{m} \partial_{x} f^{1}-\partial_{x} V \partial_{k} f^{1}\right)+F^{2}(x, \epsilon(x, k)),
$$

for any real-valued function $F^{2}$. System 3.20 - 3.21 is known as the one dimensional spherical harmonic expansion (SHE) model.

To finish this section, let us notice that, using (3.18), the diffusion coefficient (3.22) can be written as

$$
D(x, \mathrm{E})=\frac{2(\mathrm{E}-V)}{m \phi_{0}(x, \mathrm{E})},
$$

by introducing, thanks to the micro-reversibility property, the notation

$$
\phi_{0}(x, k,-k)=\phi_{0}(x, \epsilon(x, k)) .
$$

\subsection{Derivation of the one dimensional Energy-Transport model}

To derive the Energy-Transport system, we now formally perform the limit when $\beta \rightarrow 0$.

The starting point is the SHE model 3.20 - 3.21) that we write

$$
\begin{gathered}
\sqrt{\frac{2 m}{\mathrm{E}-V}}\left(\partial_{t} F^{\beta}(x, \mathrm{E})+\partial_{t} V \partial_{\mathrm{E}} F^{\beta}(x, \mathrm{E})\right)-\partial_{x} J_{S H E}^{\beta}=\frac{1}{\beta} S_{e}\left(F^{\beta}\right)(x, \mathrm{E})+S_{1}^{\alpha}\left(F^{\beta}\right)(x, \mathrm{E}), \\
J_{S H E}^{\beta}(x, \mathrm{E})=D(x, \mathrm{E}) \partial_{x} F^{\beta}(x, \mathrm{E}) .
\end{gathered}
$$


Let us introduce the Hilbert expansion

$$
F^{\beta}=F^{0}+\beta F^{1}+\ldots
$$

Injecting it into the above SHE model and identifying the terms with equal powers of $\beta$, we get

$$
\begin{gathered}
S_{e}\left(F^{0}\right)=0, \\
D S_{e}\left(F^{0}\right) \cdot F^{1}=\sqrt{\frac{2 m}{\mathrm{E}-V}}\left(\partial_{t} F^{0}+\partial_{t} V \partial_{\mathrm{E}} F^{0}\right)-\partial_{x} J_{S H E}^{0}-S_{1}^{\alpha}\left(F^{0}\right),
\end{gathered}
$$

where $D S_{e}\left(F^{0}\right) \cdot F^{1}$ denotes the Fréchet derivative of the operator $S_{e}$ at $F^{0}$ applied to $F^{1}$. We recall (see e.g. 6]) that, under the micro-reversibility assumption (3.6),

$$
S_{e}(F(\mathrm{E}))=0 \quad \Leftrightarrow \quad \exists \varphi \in \mathbb{R} \text { and } T>0, F(\mathrm{E})=\mathcal{F}_{\varphi, T}(\mathrm{E})
$$

where $\mathcal{F}_{\varphi, T}$ denotes the Fermi-Dirac distribution defined by

$$
\mathcal{F}_{\varphi, T}(\mathrm{E})=\frac{1}{\eta+e^{(\mathrm{E}-\varphi) / T}},
$$

$\eta$ being a positive constant. Thus, from (3.26), we deduce that there exist $\varphi(t, x) \in \mathbb{R}$ and $T(t, x)>0$ such that $F^{0}(t, x, \mathrm{E})=\mathcal{F}_{\varphi(t, x), T(t, x)}(\mathrm{E})$. Then, $J_{S H E}^{0}$ is given by

$$
J_{S H E}^{0}(x, \mathrm{E})=D(x, \mathrm{E}) \mathcal{F}_{\varphi, T}(\mathrm{E})\left(1-\eta \mathcal{F}_{\varphi, T}(\mathrm{E})\right) \partial_{x}\left(\frac{\varphi-\mathrm{E}}{T}\right) .
$$

Next, using the properties of the bounded non-negative self-adjoint operator $\mathcal{S}=-\sqrt{\mathrm{E}} D S_{e}(\mathcal{F})$ (see [6]), we immediately deduce from (3.27) that $F^{1}$ exists when the following expression is satisfied

$$
\int_{V}^{+\infty}\left(\begin{array}{l}
1 \\
\mathrm{E}
\end{array}\right)\left(\sqrt{\frac{2 m}{\mathrm{E}-V}}\left(\partial_{t} \mathcal{F}_{\varphi, T}+\partial_{t} V \partial_{\mathrm{E}} \mathcal{F}_{\varphi, T}\right)-\partial_{x} J_{S H E}^{0}-S_{1}^{\alpha}\left(\mathcal{F}_{\varphi, T}\right)\right) d \mathrm{E}=0 .
$$

Let us define $N_{C}$ and $N_{C}^{\mathcal{E}}$ the charge density and the energy density associated to the energy distribution 3.28 :

$$
N_{C}(t, x):=\int_{V}^{+\infty} \sqrt{\frac{2 m}{\mathrm{E}-V}} \mathcal{F}_{\varphi, T}(\mathrm{E}) d \mathrm{E} \quad \text { and } \quad N_{C}^{\mathcal{E}}(t, x):=\int_{V}^{+\infty} \mathrm{E} \sqrt{\frac{2 m}{\mathrm{E}-V}} \mathcal{F}_{\varphi, T}(\mathrm{E}) d \mathrm{E}
$$

Then, solvability condition (3.30) leads, after an integration by parts, to the EnergyTransport (ET) system :

$$
\begin{gathered}
\partial_{t} N_{C}-\partial_{x} J_{C}=0, \quad \partial_{t} N_{C}^{\mathcal{E}}-\partial_{t} V N_{C}-\partial_{x} J_{C}^{\mathcal{E}}=W(\varphi, T), \\
J_{C}=\mathbb{D}_{00} \partial_{x}\left(\frac{\varphi}{T}\right)-\mathbb{D}_{01} \partial_{x}\left(\frac{1}{T}\right), \quad J_{C}^{\mathcal{E}}=\mathbb{D}_{10} \partial_{x}\left(\frac{\varphi}{T}\right)-\mathbb{D}_{11} \partial_{x}\left(\frac{1}{T}\right),
\end{gathered}
$$

where the diffusion matrix is defined by the coefficients

$$
\mathbb{D}_{i j}(x)=\int_{V}^{+\infty} \mathrm{E}^{i+j} D(x, \mathrm{E}) \mathcal{F}_{\varphi, T}(\mathrm{E})\left(1-\eta \mathcal{F}_{\varphi, T}(\mathrm{E})\right) d \mathrm{E}, \quad 0 \leq i, j \leq 1
$$


and the relaxation term $W$ is the $\alpha^{2}$ order coefficient of

$$
W^{\alpha}(\varphi, T)=\int_{V}^{+\infty} \mathrm{E} S_{1}^{\alpha}\left(\mathcal{F}_{\varphi, T}\right)(\mathrm{E}) d \mathrm{E} .
$$

After straightforward but tedious computations, we get that $W(\varphi, T)$ is proportional to $T_{L}-T$, where $T_{L}$ is the lattice temperature. When the relaxation term is large compared to the others terms, the second equation in (3.32) implies formally that $T$ relaxes to $T_{L}$. Then, 3.32 reduces to the well-known Drift-Diffusion (DD) equation

$$
\partial_{t} N_{C}-\partial_{x}\left(\frac{\mathbb{D}_{00}}{T_{L}} \partial_{x} \varphi\right)=0
$$

\section{Interface boundary conditions}

\subsection{Derivation at the SHE level}

Following [19], we introduce the definition :

Definition 4.1 We say that $f^{\alpha}$ is an order $p$ approximation of the Boltzmann transmission problem (3.7)-(3.9), if

(i) $f^{\alpha}$ solves the Boltzmann equation (3.7) up to order $\alpha^{p}$ in the classical region $C$.

(ii) $f^{\alpha}$ satisfies the boundary condition (3.9) up to order $\alpha^{p}$ :

$$
\mathcal{B}\left(f_{1}, f_{2}\right)(p)=O\left(\alpha^{p}\right) \text {. }
$$

(iii) The flux of particles with energy $E \geq V$, given by

$$
J^{\alpha}(x, E)=\frac{1}{\alpha}\left(f^{\alpha}(x, k(x, E))-f^{\alpha}(x,-k(x, E))\right),
$$

with $k(x, E)=\sqrt{2 m(E-V)}$, is equal at the boundary up to order $\alpha^{p}$ :

$$
\begin{array}{cl}
J^{\alpha}\left(x_{1}, E\right)=J^{\alpha}\left(x_{2}, E\right)+O\left(\alpha^{p}\right), & \forall E \geq V_{1}, \\
J^{\alpha}\left(x_{2}, E\right)=0, & \text { for } E \in\left[V_{2}, V_{1}\right) .
\end{array}
$$

We have already seen in Subsection 3.2 that the Hilbert expansion

$$
f^{\alpha}(x, k)=F^{0}(x, \epsilon(x, k))+\alpha\left(F^{1}(x, \epsilon(x, k))-\lambda \partial_{x} F^{0}(x, \epsilon(x, k))\right)+\alpha^{2} f^{2}(x, k)+O\left(\alpha^{3}\right),
$$

with $F^{0}$ and $F^{1}$ introduced respectively in $(3.16)$ and $(3.19)$ and $f^{2}$ given in $(3.23)$, is a second order approximation (in $\alpha$ ) of the Boltzmann equation (3.7) away from the interfaces. Also, with the definition (4.1), we can formally deduce the expansion

$$
J^{\alpha}(x, \epsilon(x, k))=J^{1}(x, \epsilon(x, k))+\alpha J^{2}(x, \epsilon(x, k))+O\left(\alpha^{2}\right),
$$


with $J^{1}(x, \mathrm{E})=-J_{S H E}(x, \mathrm{E})($ defined in $(3.21)$ ) and

$$
J^{2}(x, \mathrm{E})=f^{2}(x, k(x, \mathrm{E}))-f^{2}(x,-k(x, \mathrm{E}))=-D(x, \mathrm{E}) \partial_{x} F^{1}(x, \mathrm{E}),
$$

thanks to the expression of $f^{2} 3.23$.

However, the boundary conditions are not a priori satisfied. In order to take into account the interface conditions, we introduce the corrector functions $\theta_{1}$ and $\theta_{2}$. These real-valued functions are defined as the solutions of the following Milne problem, widely used in the study of boundary layer correctors in kinetic theory (see e.g. [2, 20, 25, 40])

$$
\left\{\begin{array}{l}
\frac{k}{m} \frac{\partial \theta_{i}}{\partial \xi}=Q_{0}\left(\theta_{i}\right), \quad k \in \mathbb{R}, \xi \in \Omega_{i}, i=1,2 \\
\mathcal{B}\left(\theta_{1}(0, k)-\ell(k), \theta_{2}(0, k)-\ell(k)\right)=0
\end{array}\right.
$$

where $\Omega_{1}=(-\infty, 0]$ and $\Omega_{2}=[0,+\infty)$. We recall that the interface condition operator $\mathcal{B}$ is defined in $(3.8)-(3.9)$. The function $\ell$ is a function only depending on $k$ and is given by

$$
\ell(k)=\frac{\lambda(x, k)}{D(x, k)}=\frac{\operatorname{sgn}(k)}{2},
$$

where we use (3.22) for the latter equality. Adding and substracting the equations for $k$ and $-k$ in (4.6), it is clear that bounded solutions of (4.6) should satisfy $\theta_{i}(\xi, k)=$ $\theta_{i}(\xi,-k)$ and therefore belong to the kernel of $Q_{0}$. Then, $\theta_{i}$ are functions depending on the energy and they may be defined equivalently by

$$
\mathcal{B}\left(\theta_{1}\left(\epsilon\left(x_{1}, k\right)\right)-\ell(k), \theta_{2}\left(\epsilon\left(x_{2}, k\right)\right)-\ell(k)\right)=0 .
$$

Then, we have the following result :

Theorem 4.2 Let $f^{\alpha}$ be such as in (4.3), with $F^{0}$ a solution of the SHE system (3.20)(3.21). Then, $f^{\alpha}$ is a second order approximation of the Boltzmann transmission problem (3.7) - (3.9) iff $\widetilde{F}^{\alpha}:=F^{0}+\alpha F^{1}$ satisfies the boundary conditions :

$$
\begin{gathered}
D\left(x_{1}, E\right) \partial_{x} \widetilde{F}^{\alpha}\left(x_{1}, E\right)=D\left(x_{2}, E\right) \partial_{x} \widetilde{F}^{\alpha}\left(x_{2}, E\right)=: \widetilde{J}^{\alpha}(E), \\
\widetilde{F}^{\alpha}\left(x_{1}, E\right)-\widetilde{F}^{\alpha}\left(x_{2}, E\right)=\alpha \widetilde{J}^{\alpha}(E)\left(\theta_{1}(E)-\theta_{2}(E)\right),
\end{gathered}
$$

where $\theta_{1}$ and $\theta_{2}$ are the corrector functions defined in 4.8. Moreover, $\widetilde{F}^{\alpha}$ is a solution of the SHE problem up to $O\left(\alpha^{2}\right)$.

Proof. Let us start by proving that for $f^{\alpha}$ given by 4.3 , , $f^{\alpha}$ satisfies the boundary conditions 3.9 up to order $\alpha^{2}$ iff we have :

$$
\begin{array}{cl}
D\left(x_{1}, \mathrm{E}\right) \partial_{x} F^{i}\left(x_{1}, \mathrm{E}\right)=D\left(x_{2}, \mathrm{E}\right) \partial_{x} F^{i}\left(x_{2}, \mathrm{E}\right), & \forall \mathrm{E} \geq V_{1}, \quad i=0,1, \\
D\left(x_{2}, \mathrm{E}\right) \partial_{x} F^{i}\left(x_{2}, \mathrm{E}\right)=0, \quad \text { for } \mathrm{E} \in\left[V_{2}, V_{1}\right), & i=0,1, \\
F^{0}\left(x_{1}, \mathrm{E}\right)-F^{0}\left(x_{2}, \mathrm{E}\right)=0, & \forall \mathrm{E} \geq V_{1},
\end{array}
$$




$$
F^{1}\left(x_{1}, \mathrm{E}\right)-F^{1}\left(x_{2}, \mathrm{E}\right)=\overline{J_{S H E}}(\mathrm{E})\left(\theta_{1}(\mathrm{E})-\theta_{2}(\mathrm{E})\right), \quad \forall \mathrm{E} \geq V_{1},
$$

where $\theta_{1}$ and $\theta_{2}$ are defined in 4.8 and $\overline{J_{S H E}}$ is the common value of the current in $x_{1}$ and $x_{2}$ given in (4.11) for $i=0$. For that, we first notice that, by definitions of $\ell$ in (4.7) and of $J_{S H E}$ in $(3.21)$, we have

$$
\lambda(x, k) \partial_{x} F^{0}(x, \epsilon(x, k))=J_{S H E}(x, \epsilon(x, k)) \ell(k) .
$$

Then, injecting (4.3) in 3.9$)$, we get

$$
\mathcal{B}\left(F_{1}^{0}, F_{2}^{0}\right)+\alpha \mathcal{B}\left(F_{1}^{1}-J_{S H E}\left(x_{1}\right) \ell, F_{2}^{1}-J_{S H E}\left(x_{2}\right) \ell\right)=O\left(\alpha^{2}\right) .
$$

Using the reciprocity relations (2.7) and (2.9), and the fact that $\epsilon\left(x_{2},-k_{+}(k)\right)=\epsilon\left(x_{1}, k\right)$, we deduce from $(3.8)$ that $\mathcal{B}\left(F_{1}^{0}, F_{2}^{0}\right)=0$ iff

$$
\begin{array}{ll}
F^{0}\left(x_{1}, \epsilon\left(x_{1}, k\right)\right)=F^{0}\left(x_{2}, \epsilon\left(x_{1}, k\right)\right), & \text { for } k<0, \\
F^{0}\left(x_{1}, \epsilon\left(x_{2}, k\right)\right)=F^{0}\left(x_{2}, \epsilon\left(x_{2}, k\right)\right), & \text { for } k>k_{+}(0) .
\end{array}
$$

Since for $k>k_{+}(0)$, we have $\epsilon\left(x_{2}, k\right) \geq V_{1}$, we deduce that these conditions imply 4.12). For the second term of the left hand side of (4.14), we first assume the continuity of the current at the interface :

$$
J_{S H E}\left(x_{1}, \mathrm{E}\right)=J_{S H E}\left(x_{2}, \mathrm{E}\right)=\overline{J_{S H E}}(\mathrm{E}), \quad \forall \mathrm{E} \geq V_{1} .
$$

Then, the second term of the left hand side of (4.14) vanishes iff

$$
\mathcal{B}\left(F_{1}^{1}-\overline{J_{S H E}} \ell, F_{2}^{1}-\overline{J_{S H E}} \ell\right)=0 .
$$

Using the functions $\theta_{1}$ and $\theta_{2}$ introduced in (4.8), this latter equality is equivalent to impose

$$
\mathcal{B}\left(F_{1}^{1}-\overline{J_{S H E}} \theta_{1}, F_{2}^{1}-\overline{J_{S H E}} \theta_{2}\right)=0 .
$$

Since all the functions are energy dependent functions, this latter equality implies

$$
F^{1}\left(x_{1}, \mathrm{E}\right)-F^{1}\left(x_{2}, \mathrm{E}\right)=\overline{J_{S H E}}(\mathrm{E})\left(\theta_{1}(\mathrm{E})-\theta_{2}(\mathrm{E})\right), \quad \forall \mathrm{E} \geq V_{1}
$$

This proves that condition (ii) of Definition 4.1 is satisfied at second order iff (4.11) -4.13 are true. Moreover, using the expansion (4.4) and assuming (4.15), the condition (iii) of Definition 4.1 is satisfied at second order iff

$$
D\left(x_{1}, \mathrm{E}\right) \partial_{x} F^{1}\left(x_{1}, \mathrm{E}\right)=D\left(x_{2}, \mathrm{E}\right) \partial_{x} F^{1}\left(x_{2}, \mathrm{E}\right), \quad \forall, \mathrm{E} \geq V_{1} .
$$

Finally, conditions 4.11-4.13 are deduced from 4.15-4.17 -4.16.

Now, let us define

$$
F^{\alpha}(x, \epsilon(x, k))=\frac{1}{2}\left(f^{\alpha}(x, k)+f^{\alpha}(x,-k)\right) \text { and } J^{\alpha}(x, \epsilon(x, k))=\frac{1}{\alpha}\left(f^{\alpha}(x, k)-f^{\alpha}(x,-k)\right) .
$$

We formally deduce from (4.3) and (4.4) that

$$
F^{\alpha}(x, \mathrm{E})=F^{0}(x, \mathrm{E})+\alpha F^{1}(x, \mathrm{E})+O\left(\alpha^{2}\right)
$$


and

$$
J^{\alpha}(x, \mathrm{E})=-D(x, \mathrm{E}) \partial_{x}\left(F^{0}(x, \mathrm{E})+\alpha F^{1}(x, \mathrm{E})\right)+O\left(\alpha^{2}\right)
$$

Also, with these notations and using (3.11), we can rewrite the Boltzmann transport equation (3.7) as

$$
\begin{aligned}
\partial_{t} f^{\alpha}(x, k)+\frac{1}{\alpha}\left(\frac{k}{m} \partial_{x} f^{\alpha}(x, k)-\partial_{x} V \partial_{k} f^{\alpha}(x, k)\right)= & -\frac{1}{\alpha} \frac{m}{|k|} \phi_{0}(x, \epsilon(x, k)) J^{\alpha}(x, \epsilon(x, k)) \\
& +\frac{1}{\beta} Q_{e}\left(f^{\alpha}\right)+Q_{1}^{\alpha}\left(f^{\alpha}\right) .
\end{aligned}
$$

Adding this latter equation for $k$ and for $-k$ and multiplying by $m /|k|$, we get

$$
\frac{2 m}{|k|}\left(\partial_{t} F^{\alpha}+\partial_{t} V \partial_{\mathrm{E}} F^{\alpha}\right)+\partial_{x} J^{\alpha}=\frac{1}{\beta} S_{e}\left(F^{\alpha}\right)+S_{1}^{\alpha}\left(F^{\alpha}\right) .
$$

Consequently, we deduce that $\widetilde{F}^{\alpha}=F^{0}+\alpha F^{1}$ is a solution of the SHE problem up to $O\left(\alpha^{2}\right)$ with the boundary conditions $4.9-4.10$.

\subsection{Imposing continuity of the current}

We have obtained above interface conditions between the classical and the quantum regions directly thanks to an approximation of the boundary conditions for the kinetic model. Following the idea of [3], there is another way to derive boundary conditions for the SHE model by imposing continuity of the classical and quantum current at the interfaces. First, we remind that using definitions (2.3) and (2.14), we have $\epsilon\left(x_{1}, k_{1}(\mathrm{E})\right)=\mathrm{E}$ for $\mathrm{E}>V_{1}$. Also, $k_{2}(\mathrm{E})=k_{+}\left(k_{1}(\mathrm{E})\right)$. Consequently, thanks to (4.3), we have, for $\alpha \ll 1$, the approximations

$$
f^{\alpha}\left(x_{1}, k_{1}(\mathrm{E})\right) \approx \widetilde{F}^{\alpha}\left(x_{1}, \mathrm{E}\right) \quad \text { and } \quad f^{\alpha}\left(x_{2},-k_{+}\left(k_{1}(\mathrm{E})\right)\right) \approx \widetilde{F}^{\alpha}\left(x_{2}, \mathrm{E}\right) .
$$

So, we use $\widetilde{F}^{\alpha}\left(x_{1}\right.$, E) and $\widetilde{F}^{\alpha}\left(x_{2}\right.$, E) as alimentation functions in 2.15 and we obtain

$$
\mathcal{J}_{Q}(\mathrm{E})=\left(\widetilde{F}^{\alpha}\left(x_{1}, \mathrm{E}\right)-\widetilde{F}^{\alpha}\left(x_{2}, \mathrm{E}\right)\right) \mathcal{T}\left(k_{1}(\mathrm{E})\right)
$$

Therefore, imposing continuity of the rescaled current at interface, i.e.

$$
-\alpha \widetilde{J}^{\alpha}(\mathrm{E})=\mathcal{J}_{Q}(\mathrm{E})
$$

we obtain the interface condition

$$
\widetilde{F}^{\alpha}\left(x_{1}, \mathrm{E}\right)-\widetilde{F}^{\alpha}\left(x_{2}, \mathrm{E}\right)=-\alpha \frac{\widetilde{J}^{\alpha}(\mathrm{E})}{\mathcal{T}\left(k_{1}(\mathrm{E})\right)}, \quad \forall \mathrm{E} \geq V_{1},
$$

which is different from 4.10). The following proposition allows to link the two interface approaches 4.22 and 4.10 . 
Proposition 4.3 Let $\theta_{1}$ and $\theta_{2}$ be nonnegative real-valued functions satisfying 4.8. Then, for all $E \geq V_{1}$, we have

$$
\theta_{1}(E)-\theta_{2}(E)=1-\frac{1}{\mathcal{T}\left(k_{1}(E)\right)}
$$

where $k_{1}(E)=\sqrt{2 m\left(E-V_{1}\right)}$ and $\mathcal{T}$ is the transmission coefficient defined in (2.6).

Proof. Let us consider the problem 4.8). Then, for $k<0$, the interface condition writes, according to (3.8),

$$
\begin{aligned}
\theta_{1}\left(\epsilon\left(x_{1}, k\right)\right)-\ell(k) & =\mathcal{R}(-k)\left(\theta_{1}\left(\epsilon\left(x_{1},-k\right)\right)-\ell(-k)\right) \\
& +\mathcal{T}\left(-k_{+}(k)\right)\left(\theta_{2}\left(\epsilon\left(x_{2},-k_{+}(k)\right)\right)-\ell\left(-k_{+}(k)\right)\right) .
\end{aligned}
$$

From (4.7), we have $\ell(k)=-1 / 2$ for $k<0$ and $\ell(k)=1 / 2$ for $k>0$. Then, we can rewrite the latter identity as

$$
\theta_{1}\left(\epsilon\left(x_{1}, k\right)\right)+\frac{1}{2}=(1-\mathcal{T}(-k))\left(\theta_{1}\left(\epsilon\left(x_{1}, k\right)\right)-\frac{1}{2}\right)+\mathcal{T}(-k)\left(\theta_{2}\left(\epsilon\left(x_{1}, k\right)\right)+\frac{1}{2}\right),
$$

where we have used the reciprocity relations (2.7), (2.9) and the relation $\epsilon\left(x_{2},-k_{+}(k)\right)=$ $\epsilon\left(x_{1}, k\right)$. We deduce that, for $k<0$,

$$
1=\mathcal{T}(-k)\left(\theta_{2}\left(\epsilon\left(x_{1}, k\right)\right)-\theta_{1}\left(\epsilon\left(x_{1}, k\right)\right)\right)+\mathcal{T}(-k) .
$$

By the same token, we obtain for $k>0$ that

$$
1=\mathcal{T}(-k)\left(\theta_{2}\left(\epsilon\left(x_{2}, k\right)\right)-\theta_{1}\left(\epsilon\left(x_{2}, k\right)\right)\right)+\mathcal{T}(-k) .
$$

Finally, let $\mathrm{E} \geq V_{1}$ be given, we take $k=-k_{1}(\epsilon) \leq 0$ and after a change of variable, we deduce 4.23 from 4.24.

\subsection{Application to Energy-Transport and Drift-Diffusion models}

When $\beta \rightarrow 0$, we have seen in Section 3.3 that $\widetilde{F}^{\alpha}$ converges to the Fermi-Dirac distribution $\mathcal{F}_{\varphi, T}$ defined in $(3.28)$ and therefore $\widetilde{J}^{\alpha}$ converges to $D(x, \mathrm{E}) \partial_{x} \mathcal{F}_{\varphi, T}$. Therefore, condition 4.9 with 3.29 implies, for all $\mathrm{E} \geq V_{1}$, that

$$
D\left(x_{1}, \mathrm{E}\right) \mathcal{F}_{\varphi, T}\left(1-\eta \mathcal{F}_{\varphi, T}\right) \partial_{x}\left(\frac{\varphi\left(x_{1}\right)-\mathrm{E}}{T\left(x_{1}\right)}\right)=D\left(x_{2}, \mathrm{E}\right) \mathcal{F}_{\varphi, T}\left(1-\eta \mathcal{F}_{\varphi, T}\right) \partial_{x}\left(\frac{\varphi\left(x_{2}\right)-\mathrm{E}}{T\left(x_{2}\right)}\right)=: \overline{J_{S H E}^{0}}
$$

Multiplying this equation by the vector $\left(\begin{array}{l}1 \\ \mathrm{E}\end{array}\right)$ and integrating with respect to $\mathrm{E}$ leads to the conservation of the classical current :

$$
J_{C}\left(x_{1}\right)=J_{C}\left(x_{2}\right)=: \overline{J_{C}}, \quad J_{C}^{\mathcal{E}}\left(x_{1}\right)=J_{C}^{\mathcal{E}}\left(x_{2}\right)=: \overline{J_{C}^{\mathcal{E}}},
$$


where $J_{C}$ and $J_{C}^{\mathcal{E}}$ are defined in 3.33 . Secondly, the conditions 4.10 rewrites, thanks to 4.23 :

$$
\mathcal{T}\left(k_{1}(\mathrm{E})\right)\left(\mathcal{F}_{\varphi, T}\left(x_{1}, \mathrm{E}\right)-\mathcal{F}_{\varphi, T}\left(x_{2}, \mathrm{E}\right)\right)=\alpha \overline{J_{S H E}^{0}}(\mathrm{E})\left(1-\mathcal{T}\left(k_{1}(\mathrm{E})\right)\right), \quad \forall \mathrm{E} \geq V_{1} .
$$

Again, multiplying this equation by the vector $\left(\begin{array}{l}1 \\ \mathrm{E}\end{array}\right)$ and integrating with respect to $\mathrm{E}$, we obtain

$$
\int_{V_{1}}^{+\infty}\left(\mathcal{F}_{\varphi, T}\left(x_{1}, \mathrm{E}\right)-\mathcal{F}_{\varphi, T}\left(x_{2}, \mathrm{E}\right)\right) \mathcal{T}\left(k_{1}(\mathrm{E})\right)\left(\begin{array}{l}
1 \\
\mathrm{E}
\end{array}\right) d \mathrm{E}=\alpha \int_{V_{1}}^{+\infty} \overline{J_{S H E}^{0}}(\mathrm{E})\left(\begin{array}{l}
1 \\
\mathrm{E}
\end{array}\right)\left(1-\mathcal{T}\left(k_{1}(\mathrm{E})\right)\right) d \mathrm{E} .
$$

Then, the interface conditions for (ET) are given by equation (4.25)-(4.26).

The drift-diffusion system is formally obtained by taking a constant temperature $T=$ $T_{L}$, then conditions 4.25)-4.26 lead to the following interface conditions for (DD) :

$$
\begin{gathered}
\frac{D_{00}}{T_{L}} \partial_{x} \varphi\left(x_{1}\right)=\frac{D_{00}}{T_{L}} \partial_{x} \varphi\left(x_{2}\right)=: \overline{J_{C}} \\
\int_{V_{1}}^{+\infty}\left(\mathcal{F}_{\varphi, T}\left(x_{1}, \mathrm{E}\right)-\mathcal{F}_{\varphi, T}\left(x_{2}, \mathrm{E}\right)\right) \mathcal{T}\left(k_{1}(\mathrm{E})\right) d \mathrm{E}=\alpha \int_{V_{1}}^{+\infty} \overline{J_{S H E}^{0}}(\mathrm{E})\left(1-\mathcal{T}\left(k_{1}(\mathrm{E})\right)\right) d \mathrm{E}
\end{gathered}
$$

Remark 4.4 (Continuity of the current) As notice in Subsection 4.2, we can derive boundary conditions at the interface by imposing continuity of the classical and quantum current. Multiplying 4.22 by the vector $\left(\begin{array}{l}1 \\ E\end{array}\right)$ and integrating with respect to $E$, we get :

$$
\int_{V_{1}}^{+\infty}\left(\mathcal{F}_{\varphi, T}\left(x_{1}, E\right)-\mathcal{F}_{\varphi, T}\left(x_{2}, E\right)\right) \mathcal{T}\left(k_{1}(E)\right)\left(\begin{array}{l}
1 \\
E
\end{array}\right) d E=\alpha \int_{V_{1}}^{+\infty} \overline{J_{S H E}^{0}}(E)\left(\begin{array}{l}
1 \\
E
\end{array}\right) d E=\alpha\left(\frac{\overline{J_{C}}}{J_{C}^{\mathcal{E}}}\right) .
$$

Therefore the two conditions 4.26) and 4.29) are comparable when the following condition is satisfied

$$
\int_{V_{1}}^{+\infty} \overline{J_{S H E}^{0}}(E) \mathcal{T}\left(k_{1}(E)\right)\left(\begin{array}{l}
1 \\
E
\end{array}\right) d E \ll\left(\frac{\overline{J_{C}}}{J_{C}^{\mathcal{E}}}\right) .
$$

Since in the integral the most relevant energies are the lowest energies (for which the transmission coefficient is small except for the few resonant values), this condition is in general verified in applications. We will provide some numerical evidence for this issue in Section 6.

\section{$5 \quad$ Numerical hybrid approach}

\subsection{Stationary Transport - Poisson system}

We present in this Section the numerical strategy to implement the developed hybrid approach. Since we only focus on the equilibrium of the system, we present our approach 
for the stationary case; transient problems may also be investigated from a computational point of view (see e.g. [38]) but are not tackled in this paper. For the sake of completeness, we first recall the stationary system which is used in the simulations, writing it in unscaled form.

\subsubsection{Classical model}

For simplicity, we introduce the notation for the entropy variables

$$
u=\frac{\varphi}{k_{B} T} \quad \text { and } \quad v=-\frac{1}{k_{B} T},
$$

$k_{B}$ being the Boltzmann constant. Also, in the sequel, the notations $u_{1}, u_{2}, v_{1}$ and $v_{2}$ will stand for $u\left(x_{1}\right), u\left(x_{2}\right), v\left(x_{1}\right)$ and $v\left(x_{2}\right)$, respectively.

We remind that we consider the so-called parabolic band approximation. It means that the particle kinetic energy $\varepsilon$ is given by $\varepsilon(k)=\frac{\hbar^{2} k^{2}}{2 m}$, where $\hbar$ is the reduced Planck constant. For numerical purpose, we also consider that the Fermi-Dirac distribution (3.28) is approximated by the Boltzmann statistics, i.e. we choose $\eta=0$. It gives

$$
\mathcal{F}_{u, v}(\mathrm{E})=e^{u+\mathrm{E} v} .
$$

Then, the density 3.31) can be calculated explicitly in function of $u$ and $v$ and we obtain

$$
N_{C}=\left(\frac{2 m}{\pi \hbar^{2}}\right)^{1 / 2}\left(-\frac{1}{v}\right)^{1 / 2} e^{u+v V}=N_{0}\left(\frac{1}{k_{B} T_{L}}\right)^{1 / 2}\left(-\frac{1}{v}\right)^{1 / 2} e^{u+v V}
$$

where $T_{L}$ is the lattice temperature and $N_{0}=2\left(\frac{k_{B} T_{L} m}{2 \pi \hbar^{2}}\right)^{1 / 2}$ is the effective density of states.

Next, we assume that the scattering coefficient $\phi_{0}(3.25)$ is assumed to be an energy dependent function given by the following expression (see [16, 6])

$$
\phi_{0}(x, \mathrm{E})=\frac{(\mathrm{E}-V)^{\gamma}}{q \tau}\left(\frac{\hbar^{2}}{2 m}\right)^{1 / 2}
$$

where $q$ is the elementary charge and $\tau$ is the relaxation time. Typical choices for the parameter $\gamma$ are $\gamma=\frac{1}{2}$ which corresponds to the so-called Chen model [14] and $\gamma=0$, used by Lyumkis et al [31. We consider here the case $\gamma=\frac{1}{2}$. We also introduce the mobility constant $\mu$ defined by

$$
\mu=\frac{q \tau}{m} .
$$

\section{Stationary Energy-Transport system :}

Then, the stationary ET system writes

$$
\begin{gathered}
\partial_{x} J_{C}(u, v)=0, \\
-\frac{1}{q} \partial_{x} J_{C}^{\mathcal{E}}(u, v)=W(u, v),
\end{gathered}
$$

with

$$
J_{C}(u, v)=-\left(\mathbb{D}_{00} \partial_{x} u+\mathbb{D}_{01} \partial_{x} v\right) \quad \text { and } \quad J_{C}^{\mathcal{E}}(u, v)=-\left(\mathbb{D}_{10} \partial_{x} u+\mathbb{D}_{11} \partial_{x} v\right) .
$$


The diffusion coefficients $\mathbb{D}_{i j}$ (3.34) are given by

$$
\mathbb{D}_{i j}=\mu \frac{2}{\pi}\left(\frac{2 m}{\hbar^{2}}\right)^{1 / 2} e^{u+v V} \int_{0}^{+\infty}(\varepsilon+V)^{i+j} \sqrt{\varepsilon} e^{\varepsilon v} d \varepsilon
$$

After calculations, we obtain the following explicit expressions

$$
\mathbb{D}_{00}=\mu\left(\frac{2 m}{\pi \hbar^{2}}\right)^{1 / 2}\left(-\frac{1}{v}\right)^{3 / 2} e^{u+v V}=-\frac{\mu N_{C}}{v}
$$

where the expression of $N_{C}$ is given in (5.3);

$$
\mathbb{D}_{01}=\mathbb{D}_{10}=\mathbb{D}_{00}\left(-\frac{3}{2 v}+V\right) \quad \text { and } \quad \mathbb{D}_{11}=\mathbb{D}_{00}\left(\frac{15}{4 v^{2}}-\frac{3 V}{v}+V^{2}\right)
$$

Finally, we can show that the relaxation term $W$ is given by

$$
W(u, v)=\frac{3}{2 \tau} N_{C}\left(\frac{1}{v}+k_{B} T_{L}\right) .
$$

Boundary conditions at $x=0$ and $x=L$ :

The ET system (5.5)-(5.6) is completed with Dirichlet conditions on $u$ and $v$. We assume that the temperature is the lattice temperature at the boundaries. It gives

$$
v(0)=v(L)=-\frac{1}{k_{B} T_{L}}
$$

We also impose quasi-neutrality between the density $N_{C}$ and the given doping density $N_{D}$. It gives

$$
u(x)=\log \left(\frac{N_{D}(x)}{N_{0}}\right)+\frac{V(x)}{k_{B} T_{L}}, \quad \text { for } x \in\{0, L\} .
$$

\section{Stationary Drift-Diffusion system :}

When the temperature is constant $\left(T=T_{L}\right)$, the ET system simplifies to the DD equation

$$
\partial_{x} J_{C}=0, \quad \text { with } \quad J_{C}=-\mu N_{C} \partial_{x} \varphi
$$

where the classical density $N_{C}$ is given by

$$
N_{C}=N_{0} e^{(\varphi-V) /\left(k_{B} T_{L}\right)}
$$

\subsubsection{Quantum model}

For each wave vector $k$, we consider the following scattering states Schrödinger equation in the quantum domain $Q=\left[x_{1}, x_{2}\right]$

$$
-\frac{\hbar^{2}}{2 m} \partial_{x x} \psi_{k}(x)+V(x) \psi_{k}(x)=\epsilon(k) \psi_{k}(x), \text { with } \epsilon(k)= \begin{cases}\epsilon_{1}(k)=\varepsilon(k)+V_{1} & \text { if } k>0 \\ \epsilon_{2}(k)=\varepsilon(k)+V_{2} & \text { if } k<0\end{cases}
$$


with TBCs (2.4)-(2.5) written in unscaled form. The quantum density $N_{Q}$ is given in (2.11) where we choose the distribution function $f$ to be the normalized Boltzmann statistics defined by

$$
f\left(x_{1}, k\right)=\tilde{N} e^{V_{1} v_{1}} e^{\varepsilon(k) v_{1}+u_{1}} \quad \text { for } k>0, \quad f\left(x_{2}, k\right)=\widetilde{N} e^{V_{2} v_{2}} e^{\varepsilon(k) v_{2}+u_{2}} \quad \text { for } k<0 .
$$

In this expression $\widetilde{N}$ is a normalization coefficient such that

$$
\widetilde{N}\left(\int_{k>0} e^{\varepsilon(k) v_{1}} d k+\int_{k<0} e^{\varepsilon(k) v_{2}} d k\right)=N_{0} .
$$

After straightforward calculations it gives :

$$
\tilde{N}=N_{0}\left(\frac{\hbar^{2}}{2 \pi m}\right)^{1 / 2}\left(\frac{\sqrt{v_{1}}+\sqrt{v_{2}}}{2}\right)
$$

Finally, as discussed in Lemma 2.1, the quantum particle current can be written in the following form

$$
J_{Q}=\frac{q \hbar}{m} \widetilde{N}\left(e^{u_{1}} \int_{0}^{+\infty} k \mathcal{T}(k) e^{\epsilon_{1}(k) v_{1}} d k-e^{u_{2}} \int_{0}^{+\infty} k \mathcal{T}(k) e^{\epsilon_{1}(k) v_{2}} d k\right)
$$

where $\mathcal{T}$ are the transmission coefficients defined in $(2.6)$. Analogously, we also define the quantum energy current by

$$
J_{Q}^{\mathcal{E}}=\frac{q \hbar}{m} \widetilde{N}\left(e^{u_{1}} \int_{0}^{+\infty} k \epsilon_{1}(k) \mathcal{T}(k) e^{\epsilon_{1}(k) v_{1}} d k-e^{u_{2}} \int_{0}^{+\infty} k \epsilon_{1}(k) \mathcal{T}(k) e^{\epsilon_{1}(k) v_{2}} d k\right) .
$$

\subsubsection{Interface conditions}

Now we describe the interface conditions in $x_{1}$ and $x_{2}$ that complete the hybrid Schrödinger Energy-Transport (S-ET) model and the hybrid Schrödinger Drift-Diffusion (S-DD) model. As explained in Section 4, these connection conditions can be obtained computing boundary layer corrections from the kinetic model (approaches denoted S-ET1 and S-DD1 in the sequel) or preserving the continuity of the current (approaches denoted S-ET2 and S-DD2 in the sequel).

For the ET system, the conservation of classical currents 4.25 is completed with 4.26 for S-ET1 or 4.29 for S-ET2. It gives

$$
J_{Q}=\Lambda \overline{J_{C}}, \quad J_{Q}^{\mathcal{E}}=\Lambda^{\mathcal{E}} \overline{J_{C}^{\mathcal{E}}}
$$

where $\Lambda=\Lambda^{\mathcal{E}}=1$ for S-ET2, instead the following explicit expressions hold for S-ET1 :

$$
\begin{gathered}
\Lambda=1-\frac{\partial_{x} u_{1} \int_{0}^{+\infty} \mathcal{T}(k) k^{2} e^{\varepsilon(k) v_{1}} d k+\partial_{x} v_{1} \int_{0}^{+\infty} \mathcal{T}(k) k^{2}\left(\varepsilon(k)+V_{1}\right) e^{\varepsilon(k) v_{1}} d k}{\partial_{x} u_{1} \int_{0}^{+\infty} k^{2} e^{\varepsilon(k) v_{1}} d k+\partial_{x} v_{1} \int_{0}^{+\infty} k^{2}\left(\varepsilon(k)+V_{1}\right) e^{\varepsilon(k) v_{1}} d k}, \\
\Lambda^{\mathcal{E}}=1-\frac{\partial_{x} u_{1} \int_{0}^{+\infty} \mathcal{T}(k) k^{2}\left(\varepsilon(k)+V_{1}\right) e^{\varepsilon(k) v_{1}} d k+\partial_{x} v_{1} \int_{0}^{+\infty} \mathcal{T}(k) k^{2}\left(\varepsilon(k)+V_{1}\right)^{2} e^{\varepsilon(k) v_{1}} d k}{\partial_{x} u_{1} \int_{0}^{+\infty} k^{2}\left(\varepsilon(k)+V_{1}\right) e^{\varepsilon(k) v_{1}} d k+\partial_{x} v_{1} \int_{0}^{+\infty} k^{2}\left(\varepsilon(k)+V_{1}\right)^{2} e^{\varepsilon(k) v_{1}} d k} .
\end{gathered}
$$


To obtain these expressions, we use the explicit formulations of $J_{C}, J_{C}^{\mathcal{E}}$ and $J_{S H E}^{0}$ (coming from (5.7) and (3.29) ), and the fact that (4.26) can be also written (in rescaled variables)

$$
\left(\begin{array}{l}
J_{Q} \\
J_{Q}^{\mathcal{E}}
\end{array}\right)=\left(\begin{array}{l}
\overline{J_{C}}\left(1-\frac{1}{\overline{J_{C}}} \int_{V_{1}}^{+\infty} \overline{J_{S H E}^{0}}(\mathrm{E}) \mathcal{T}\left(k_{1}(\mathrm{E})\right) d \mathrm{E}\right) \\
\overline{J_{C}^{\mathcal{E}}}\left(1-\frac{1}{\overline{J_{C}^{\varepsilon}}} \int_{V_{1}}^{+\infty} \overline{J_{S H E}^{0}}(\mathrm{E}) \mathcal{T}\left(k_{1}(\mathrm{E})\right) \mathrm{E} d \mathrm{E}\right)
\end{array}\right) .
$$

For the $D D$ system, since the temperature is constant, conditions (5.18) are reduced to

$$
J_{Q}=\Lambda \overline{J_{C}},
$$

with $\Lambda=1$ for S-DD2 and with the following explicit expression for S-DD1

$$
\Lambda=1-\frac{\int_{0}^{+\infty} \mathcal{T}(k) k^{2} e^{-\varepsilon(k) /\left(k_{B} T_{L}\right)} d k}{\int_{0}^{+\infty} k^{2} e^{-\varepsilon(k) /\left(k_{B} T_{L}\right)} d k} .
$$

Therefore, the derivation of a hierarchy of models (passing through the SHE model) allow us to obtain an explicit expression of the coupling conditions. Instead, in [15, several approximate expressions of the so-called extrapolation coefficient were obtained. We remark that (5.22) is highly related to the Marshak approximation proposed in Section 2.4.2 of [15].

\subsubsection{Self-consistent formulation}

Finally, we are interested in self-consistent computations. The transport equations are coupled with the following Poisson equation for the electrostatic potential $V_{P}$

$$
-\nabla\left(\epsilon_{r} \nabla V_{P}(x)\right)=\frac{q}{\epsilon_{0}}\left(N_{D}(x)-N(x)\right), \quad x \in(0, L) .
$$

$\epsilon_{0}$ is the permittivity in vacuum, $\epsilon_{r}$ the relative permittivity, $N_{D}$ the prescribed doping density and $N$ the hybrid charge density defined by

$$
N(x)= \begin{cases}N_{Q}(x) & \text { for } x \in Q, \\ N_{C}(x) & \text { for } x \in C,\end{cases}
$$

where $N_{Q}$ and $N_{C}$ are given in (2.11) and (5.3), respectively. Notice that $V$ in (5.15) or (5.5)-(5.6) is given by $V=-q V_{P}$. Equation (5.23) is supplemented by the Dirichlet boundary conditions $V_{P}(0)=0$ and $V_{P}(L)=V_{a}$ in order to impose an applied voltage $V_{a}$.

\subsection{Algorithm}

We describe here the numerical issues related to the solution of the coupled hybrid transport equations with the Poisson equation. We first consider the whole system for zero applied voltage $\left(V_{a}=0\right)$ and then we increment $V_{a}$ step by step. Because of the highly nonlinear coupling between the density and the potential equations, we use an iterative method of Gummel type [26]. The different steps of one iteration are detailed here. 
1. Let $V_{P}^{\text {old }}$ be a given electrostatic potential. We define the potential energy $V=$ $-q V_{P}^{\text {old }}$.

2. For each wave vector $k$, we solve in $Q$ the Schrödinger equation (5.15) with TBCs. It is transformed to an initial value problem and discretized with a Crank-Nicolson scheme (see 8 for details). Then, the transmission coefficients $\mathcal{T}$ are obtained.

3. We solve the stationary classical model in $C$ : either the ET system (5.5)-(5.6) with the boundary conditions (5.11)-5.12) and the connection conditions (4.25)-(5.18), or the DD equation (5.13) with (5.12)-(4.27)-(5.21). These classical equations are discretized by means of a mixed finite element scheme of lowest order in hybridized form [1]. More precisely, we provide in the following a brief description of this approach in the simply connected region $\left[0, x_{1}\right]$. We introduce the notations

$$
U=(u, v)^{T}, \quad \mathbb{J}=\left(J_{C}, J_{C}^{\mathcal{E}}\right)^{T}, \quad W(U)=(0,-q W(u, v)) \text { and } \mathbb{D}=\left(\begin{array}{cc}
\mathbb{D}_{00} & \mathbb{D}_{01} \\
\mathbb{D}_{10} & \mathbb{D}_{11}
\end{array}\right) .
$$

Then, the system (5.5)-(5.6) can be written in compact form as

$$
\mathbb{J}=-\mathbb{D}(U) \partial_{x} U, \quad \partial_{x} \mathbb{J}=W(U) .
$$

Let us introduce a partition of $\left[0, x_{1}\right]$ with nodes $z_{i}, i=0, \ldots, N_{x_{1}}$. Denoting by $U_{i}$ an approximation of $U\left(x_{i}\right)$, we take the piecewise constant approximation of $U$ given, in the interval $I_{i}:=\left(z_{i-1}, z_{i}\right)\left(i=1, \ldots, N_{x_{1}}\right)$, by

$$
\bar{U}_{i}=\frac{U_{i-1}+U_{i}}{2},
$$

and define piecewise constant diffusion coefficients as $\mathbb{D}_{k l}\left(\bar{U}_{i}\right)($ for $k, l=0,1)$ and a piecewise constant relaxation term as $W\left(\bar{U}_{i}\right)$.

To use a mixed finite element discretization of lowest order in hybridized form [1] (see also [22, 23, 34] for applications to ET), we introduce the following finite dimensional spaces :

$$
\begin{aligned}
X_{h} & =\left\{\sigma \in L^{2}((0, L)): \sigma(x)=a_{i}+b_{i}\left(x-z_{B_{i}}\right) \text { in } I_{i}, i=1, \ldots, N_{x_{1}}\right\} \\
Y_{h} & =\left\{\xi \in L^{2}((0, L)): \xi \text { is constant in } I_{i}, i=1, \ldots, N_{x_{1}}\right\} \\
Z_{h, \chi} & =\left\{q=\left(q_{i}\right), i=0, \ldots, N_{x_{1}}, \text { s.t. } q_{0}=\chi(0), q_{N_{x_{1}}}=\chi\left(x_{1}\right)\right\}
\end{aligned}
$$

where $z_{B_{i}}=\frac{1}{2}\left(z_{i-1}+z_{i}\right)$ denotes the central point of the interval $I_{i}$, and $\chi$ is prescribed.

Then, the mixed-hybrid formulation of (5.24) reads as follows: Find $\mathbb{J}_{h} \in X_{h}^{2}$, $P_{h} \in Y_{h}^{2}$, and $U_{h} \in Z_{h, u_{b}} \times Z_{h, v_{b}}$ (where $u_{b}$ and $v_{b}$ are the boundary values of $u$ and 
$v$ ) such that

$$
\begin{aligned}
\sum_{i=1}^{N_{x_{1}}}\left(\int_{I_{i}} \phi_{h} \cdot \mathbb{D}\left(\bar{U}_{i}\right)^{-1} \mathbb{J}_{h} d x+\int_{I_{i}} P_{h} \cdot \nabla_{x} \phi_{h} d x-\left[\phi_{h} \cdot U_{h}\right]_{z_{i-1}}^{z_{i}}\right) & =0, \\
\sum_{i=1}^{N_{x_{1}}}\left(\int_{I_{i}} \Psi_{h} \cdot \nabla_{x} \mathbb{J}_{h} d x-\int_{I_{i}} W\left(\bar{U}_{i}\right) \cdot \Psi_{h} d x\right) & =0, \\
-\sum_{i=1}^{N_{x_{1}}}\left[\mu_{h} \cdot \mathbb{J}_{h}\right]_{z_{i-1}}^{z_{i}} & =0
\end{aligned}
$$

for all $\phi_{h} \in X_{h}^{2}, \Psi_{h} \in Y_{h}^{2}$, and $\mu_{h} \in Z_{h .0}^{2}$. Equation (5.25) is derived from the weak formulation of the first equations in (5.24); (5.26) comes from the weak form of the second equations in (5.24); and (5.27) imposes the continuity of the currents at the nodes. Finally, since we are dealing with an interface problem, the values of $u_{b}$ and $v_{b}$ in $x_{1}$ are not a priori given but are defined through the conditions (4.25)-(5.18), and clearly the two classical regions $\left[0, x_{1}\right]$ and $\left[x_{2}, L\right]$ cannot be treated separately.

Thanks to the discontinuity of the spaces $X_{h}$ and $Y_{h}$, we can apply static condensation in order to reduce the size of the discrete system and obtain an algebraic system for the variable $U_{h}$ only. More precisely, choosing first the test functions

$$
\phi_{h}=\left\{\begin{array}{ll}
(1,0)^{T} & \text { in } I_{i} \\
(0,0)^{T} & \text { elsewhere }
\end{array} \text { and } \phi_{h}= \begin{cases}(0,1)^{T} & \text { in } I_{i} \\
(0,0)^{T} & \text { elsewhere }\end{cases}\right.
$$

in (5.25) and then, analogously choosing $\Psi_{h}$ in (5.26), we obtain the piecewise linear (discrete) current

$$
\mathbb{J}_{h \mid I_{i}}=\mathbb{D}\left(\bar{U}_{i}\right) \frac{U_{i}-U_{i-1}}{z_{i}-z_{i-1}}+W\left(\bar{U}_{i}\right)\left(x-z_{B_{i}}\right) .
$$

Imposing continuity at the nodes (through (5.27)) we obtain

$$
\begin{aligned}
\frac{\mathbb{D}\left(\bar{U}_{i}\right)}{z_{i}-z_{i-1}} U_{i-1}-\left(\frac{\mathbb{D}\left(\bar{U}_{i}\right)}{z_{i}-z_{i-1}}\right. & \left.+\frac{\mathbb{D}\left(\bar{U}_{i+1}\right)}{z_{i+1}-z_{i}}\right) U_{i}+\frac{\mathbb{D}\left(\bar{U}_{i+1}\right)}{z_{i+1}-z_{i}} U_{i+1} \\
& =\frac{1}{2}\left(\left(z_{i}-z_{i-1}\right) W\left(\bar{U}_{i}\right)+\left(z_{i+1}-z_{i}\right) W\left(\bar{U}_{i+1}\right)\right) .
\end{aligned}
$$

for $i=1, \ldots, N_{x_{1}}-1$. We point out explicitly that, since the first component of $W(U)$ is null, the approximation of the current $J_{0}$ is piecewise constant (see (5.28) and that, thanks to (5.27), it is indeed globally constant. The full system (made of (5.29), the analogous equations for the region $\left[x_{2}, L\right]$ and the two interfaces conditions) forms a non-linear system that is solved using a Newton algorithm.

4. We compute the classical density $N_{C}$ (5.3) or (5.14), and the quantum density $N_{Q}$ 2.11) using $u$ and $v$ at boundaries $x_{1}$ and $x_{2}$. 
5. We solve the Poisson equation (5.23) using finite differences, modified according to the Gummel iteration algorithm, that is

$$
-\nabla\left(\epsilon_{r} \nabla V_{P}^{\text {new }}\right)=\frac{q}{\epsilon_{0}} N_{D}-\frac{q}{\epsilon_{0}} N\left[V_{P}^{\text {old }}\right]\left(1+\frac{q}{k_{B} T_{L}}\left(V_{P}^{\text {new }}-V_{P}^{\text {old }}\right)\right), \text { in }(0, L) .
$$

6. We repeat the steps until the quantity $\left\|V_{P}^{\text {old }}-V_{P}^{\text {new }}\right\|_{L^{\infty}}$ becomes sufficiently small. Once the convergence is reached, we increment $V_{a}$ and start a new iteration.

\section{$6 \quad$ Numerical results}

In this section, we propose numerical simulations of a one-dimensional resonant tunneling diode in order to test the interface conditions described previously. We choose the same geometry and data as in [21]. The device (see Figure 1) has a total length of $135 \mathrm{~nm}$. It is made of two highly doped GaAs regions $\left(N_{D}^{+}=10^{24} \mathrm{~m}^{-3}\right)$ of $50 \mathrm{~nm}$ at extremities, surrounding an active zone of $35 \mathrm{~nm}$ with smaller doping $\left(N_{D}^{-}=5.10^{21} \mathrm{~m}^{-3}\right)$. This active zone contains a quantum well of $5 \mathrm{~nm}$ length sandwiched between two $5 \mathrm{~nm}$ AlGaAs barriers placed between two $10 \mathrm{~nm}$ GaAs spacer layer. It is in this small region that the important physical effects such as tunneling or scattering take place.

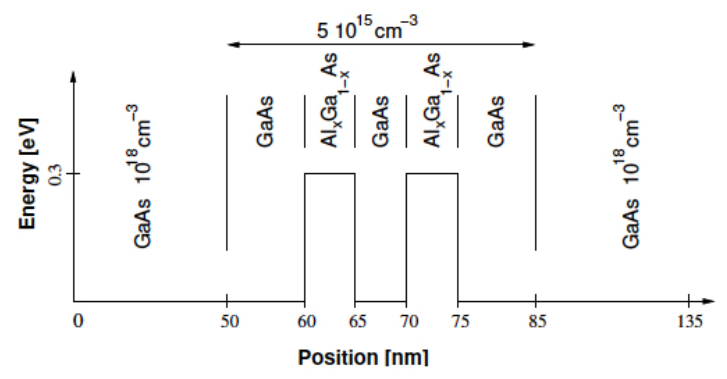

Figure 1: Geometry of the resonant tunneling diode and profile of the double barrier (taken from [21]).

The physical parameters are chosen as follows. Simulations are done at room temperature $T_{L}=300 \mathrm{~K}$. The double barrier height is of $V_{b a r}=0.3 \mathrm{eV}$. However, for numerical purposes, we will also consider the case in which $V_{b a r}=0.15 \mathrm{eV}$. The physical effect of the double barrier is a shift in the quasi-Fermi energy that we model by an additional function added to the electrostatic potential. The electron effective mass $m$ (relative to the vacuum electron mass) is 0.067 . The relative semiconductor permittivity $\epsilon_{r}$ is constant throughout the structure and equal to 11.44. The relaxation time $\tau$ is $10^{-12} \mathrm{~s}$, that gives a mobility constant $\mu$ defined in (5.4) equal to $2.63 \mathrm{~m}^{2} \cdot \mathrm{V}^{-1} \cdot \mathrm{s}^{-1}$. Finally, in order to obtain a physical density (expressed in $\mathrm{m}^{-3}$ ), we rescale the one dimensional density $N$ by the factor $\frac{m k_{B} T_{L}}{2 \pi \hbar^{2}}$. The same rescaling is used for the current to obtain a quantity expressed in A.m $m^{-2}$.

The mesh size is $h=0.1 \mathrm{~nm}$ both in the quantum zone and in the classical zones. The same discretization is used to solve the transport system and the Poisson equation. 
Due to resonant energies, we compute the scattering states of the Schrödinger equation for each wave vector $k$ being in a highly refined uniform grid $\left(10^{5}\right.$ values $)$ in order to take into account the contribution of each significant energy. An adaptive algorithm (as proposed in [35]) would be probably more appropriate. Finally, for the applied bias at extremities of the device, we numerically found that an increment step equal to $0.02 \mathrm{~V}$ is a reasonable choice, except close to the current peak where a step of $2.10^{-3} \mathrm{~V}$ is used.

For the numerical results presented in this paper, the classical-quantum interfaces are placed exactly at the doping junctions (50 and $85 \mathrm{~nm}$ ) except for Figures 6 and 7 .

\subsection{Results for the case $V_{b a r}=0.3 \mathrm{eV}$}

We display here the numerical results obtained thanks to the algorithm proposed in Section 5.2, for a potential barrier $V_{b a r}=0.3 \mathrm{eV}$. First, in Figure 2, we plot the output Current-Voltage characteristics. We recall that the notations S-ET1 and S-ET2 refer to the Energy-Transport model with the two different interface condition approaches (see Section 5.1.3.

The shape of the current is typical of resonant tunneling diodes (see e.g. [35, 15, 21]) with a peak due to electrons having the resonant energies of the double barrier. Zooms of these current-voltage characteristics are displayed in Figure 3 and the difference of these two curves are presented in Figure 4. A close inspection of (5.18) with the different values of $\Lambda$ and $\Lambda^{\mathcal{E}}\left(\Lambda=\Lambda^{\mathcal{E}}=1\right.$ for S-ET2 and $\Lambda$ and $\Lambda^{\mathcal{E}}$ given by (5.19)-(5.20) for S-ET1) shows that the difference is directly linked to the evolution of the transmission coefficients $\mathcal{T}(k)$ and a larger difference is expected for large applied bias. However, this difference is small and we may deduce that both approaches give very similar results. It has already been noted for the Drift-Diffusion system in [3]. It confirms that the interface conditions derived by imposing exact continuity of the current (much easier to implement) give a good approximation to the interface conditions obtained by computation of an approximation of the boundary layers.

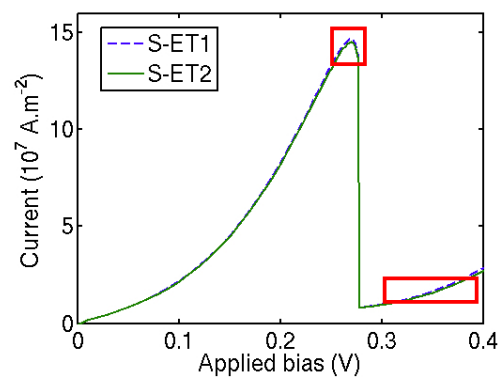

Figure 2: Current-Voltage characteristics obtained for the S-ET model with the two interface condition approaches $\left(V_{\text {bar }}=0.3 \mathrm{eV}\right)$.

This behavior is confirmed with Figure 5 that represents the potential energy and the density within the device for an applied voltage of $0.2 \mathrm{~V}$. Moreover, we emphasize that the two coupling approaches do not enforce density continuity at interfaces, as observed 

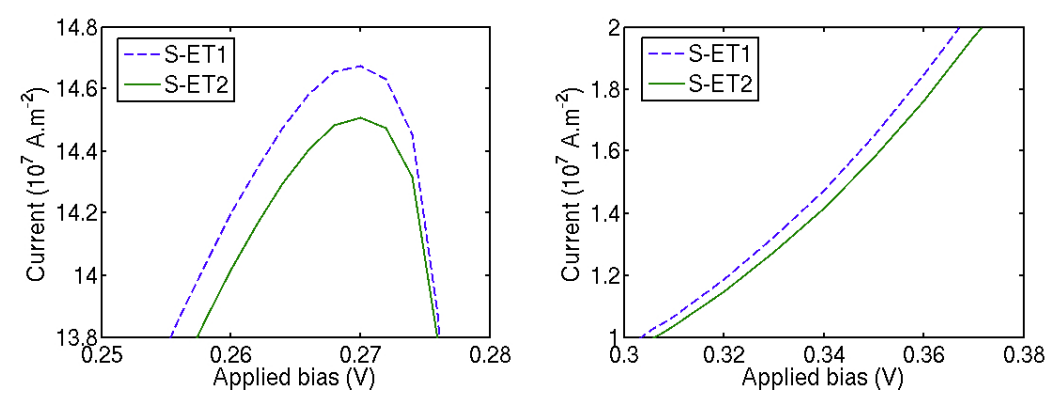

Figure 3: Zoom of the Current-Voltage characteristics around the peak (left) and after the peak (right).

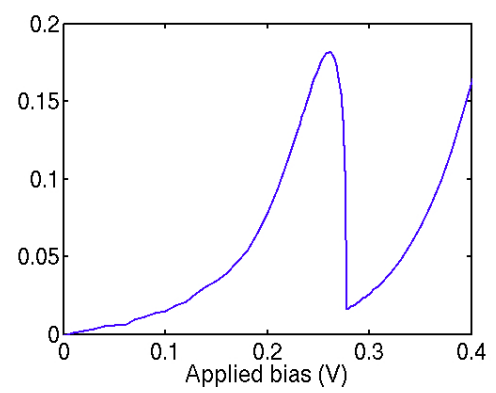

Figure 4: Difference between the two Current-Voltage characteristics of Figure 2.

in the right picture of Figure 5. It is also the case for the two densities presented in Figure 6 where the position of the interfaces has been moved. Usually, the interfaces are placed exactly at the doping junctions $\left(x_{1}=50 \mathrm{~nm}\right.$ and $\left.x_{2}=85 \mathrm{~nm}\right)$. In Figure 6, in the left picture the interfaces are moved ouside the channel $\left(x_{1}=45 \mathrm{~nm}\right.$ and $\left.x_{2}=90 \mathrm{~nm}\right)$ whereas in the right picture the interfaces are moved inside the channel $\left(x_{1}=55 \mathrm{~nm}\right.$ and $\left.x_{2}=80 \mathrm{~nm}\right)$.
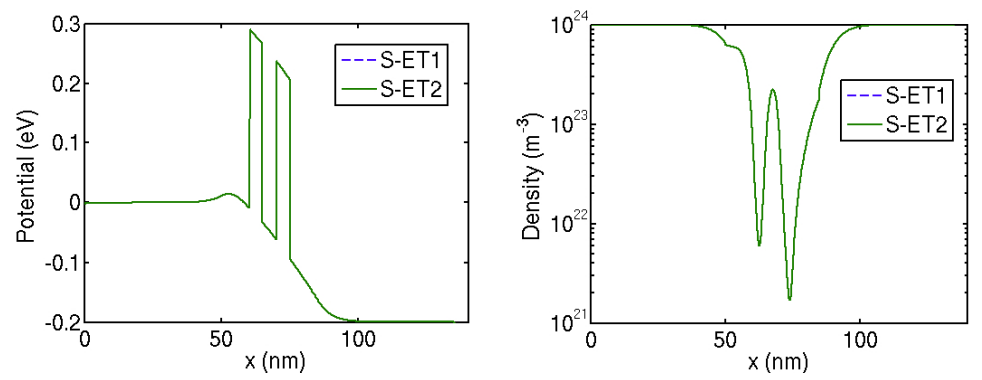

Figure 5: Left: Potential energy $(\mathrm{eV})$; Right: density $\left(\mathrm{m}^{-3}\right)$ obtained for $V_{a}=0.2 \mathrm{~V}$.

Finally, in Figure 7, we present the Current-Voltage characteristics obtained with different interface positions. Since, we observe the same results for the two interface condition approaches, we present only those obtained with S-ET2. In the left picture of 

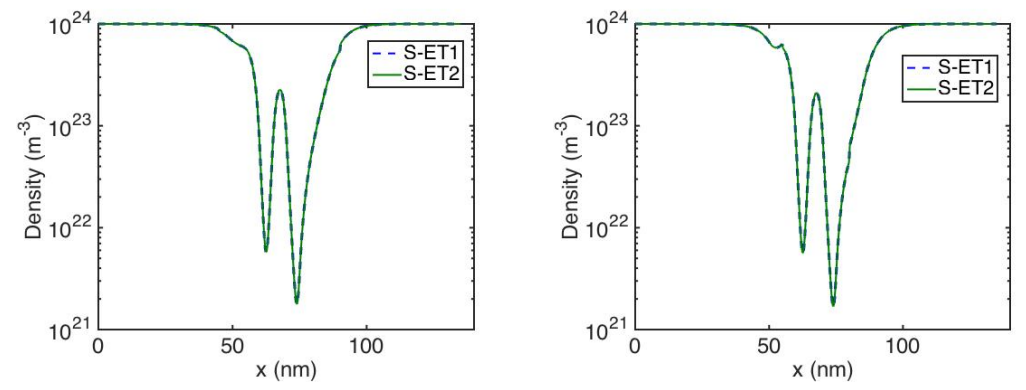

Figure 6: Density $\left(\mathrm{m}^{-3}\right)$ obtained for $V_{a}=0.2 \mathrm{~V}$ with $x_{1}=45 \mathrm{~nm}$ and $x_{2}=90 \mathrm{~nm}$ (left) and with $x_{1}=55 \mathrm{~nm}$ and $x_{2}=80 \mathrm{~nm}$ (right).

Figure 7, the position of the right interface is fixed $\left(x_{2}=85 \mathrm{~nm}\right)$ whereas the position of the left interface $x_{1}$ is moved. In the right picture, it is the contrary $\left(x_{1}=55 \mathrm{~nm}\right.$ and $x_{2}$ variable). The solid lines correspond to the usual interface positions (doping junctions), the dashed lines to an interface placed outside the channel and the dashdotted lines to an interface placed inside the channel. As reported in [15, 3, 21, the interface position affects the Current-Voltage characteristics and the influence of the right interface position $x_{2}$ is less strong than that of $x_{1}$. It is due to the fact that in the drain there are essentially high energetic particles which are equally well described by the Energy-Transport or the Schrödinger model.
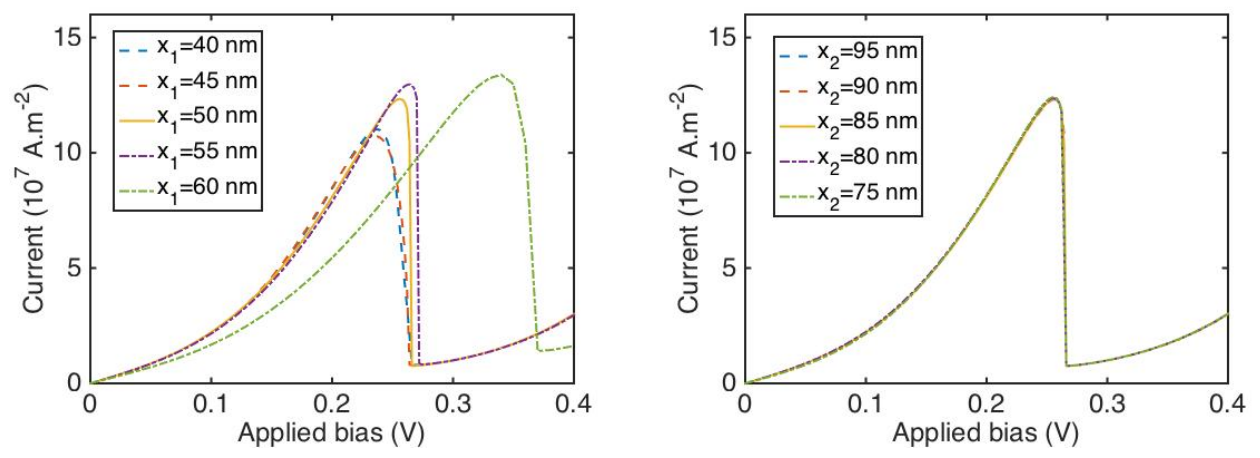

Figure 7: Current-Voltage characteristics obtained moving the left interface position $x_{1}$ (left) and moving the right interface position $x_{2}$ (right).

\subsection{Results for the case $V_{b a r}=0.15 \mathrm{eV}$}

In the previous case, we observed minor differences between the two approaches that we use to impose the interface conditions. In order to magnify the difference between these two approaches, we present now simulations for a less physically significant case, dividing the double barrier height by $2\left(V_{b a r}=0.15 \mathrm{eV}\right)$. 
In Figure 8, we represent the logarithm of the transmission coefficient $\mathcal{T}$ within the device as a function of the energy, for an applied voltage $V_{a}=0.2 \mathrm{~V}$ and for the two potential barriers $\left(V_{b a r}=0.3 \mathrm{eV}\right.$ and $\left.V_{b a r}=0.15 \mathrm{eV}\right)$. We clearly observe that the transmission coefficient is more important with a smaller barrier. A consequence is that the correction coefficients $\Lambda$ and $\Lambda^{\mathcal{E}}(5.19)-(5.20)$ get away from 1. Therefore, the output Current-Voltage characteristics are slightly different for the two interface condition approaches (Figure 9), specially after the peak. Notice however that the physical informations such as the peak location and the peak-to-valley height are stable. Finally, Figure 10 represents the temperature inside the classical zones for two applied voltages. Some perceptible differences are observed. However, we would like to emphasize that the two approaches give similar quantities (including the current) inside the quantum zone.

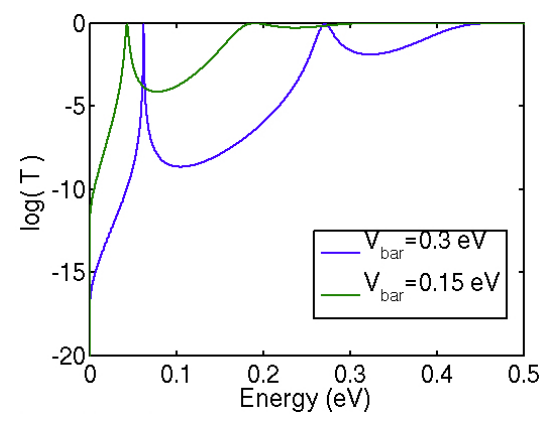

Figure 8: Transmission coefficient for $V_{a}=0.2 \mathrm{~V}$.

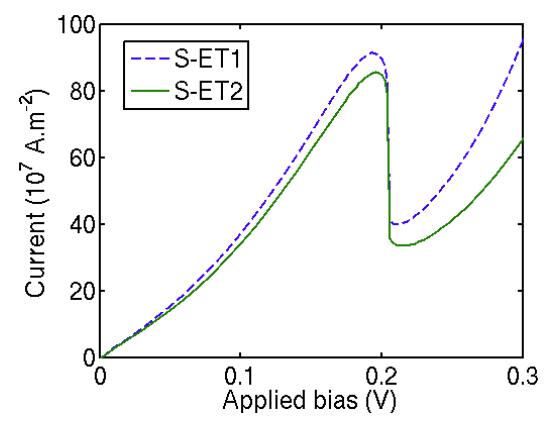

Figure 9: Current-Voltage characteristics obtained for the S-ET model with the two interface condition approaches $\left(V_{b a r}=0.3 \mathrm{eV}\right)$.

To finish, it is interesting to observe the behaviour of the algorithm when letting the relaxation time $\tau$ going to 0 . In fact, as it has been noticed in the previous sections, the ET system relaxes towards the DD system. In Figure 11, we display the numerical results obtained for the S-DD1 system and for the S-ET1 system with a relaxation time going to 0 . As expected the curves computed with the S-ET1 system converge towards the one computed for the S-DD1. We recover the same behavior with S-ET2 and S-DD2 (as well as for the two approaches in the case $V_{b a r}=0.3 \mathrm{eV}$ ), except that in these cases the variations between the two models (S-DD and S-ET) are much smaller. 

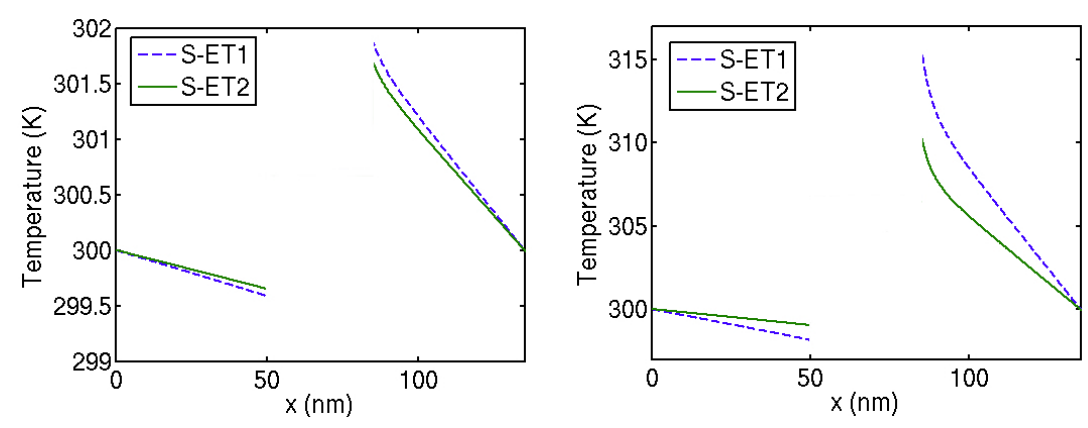

Figure 10: Temperature in the classical zones for $V_{a}=0.1 \mathrm{~V}$ (left) and $V_{a}=0.4 \mathrm{~V}$ (right).
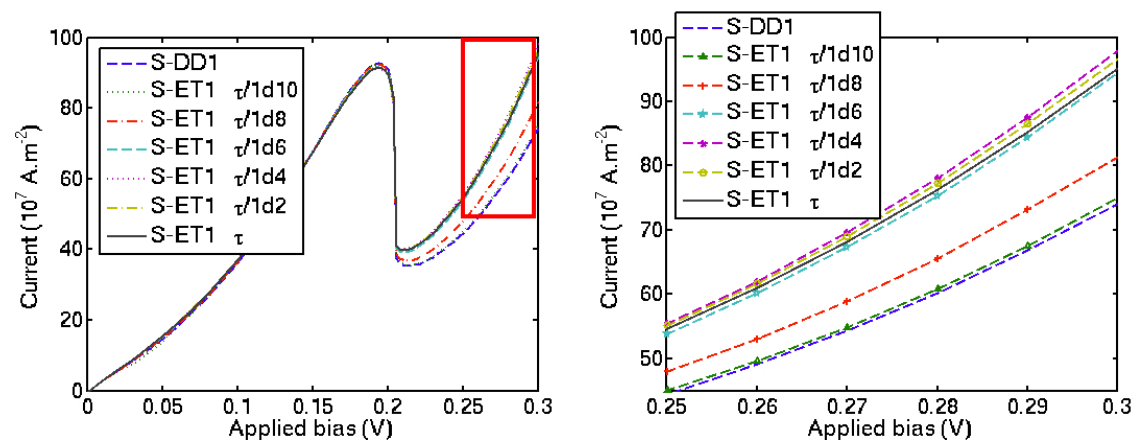

Figure 11: Current-Voltage characteristics in function of the relaxation time $\tau$. The figure on the right is a zoom for high values of the applied bias.

\section{Conclusion}

We have proposed in this paper strategies for hybrid classical-quantum transport. These strategies are applied to devices for which quantum effects are well localized in a small region of the device. More precisely, a quantum region $Q=\left[x_{1}, x_{2}\right]$ is sandwiched between two classical regions $C=\left[0, x_{1}\right] \cup\left[x_{2}, L\right]$. The classical region is assumed to be highly collisional and diffusive approximation of the Boltzmann transport equation is considered leading to Energy-Transport or Drift-Diffusion equations. Then, we have considered interface conditions to couple the Schrödinger equation with Energy-Transport. After relaxation towards the Drift-Diffusion system, we recover the same boundary conditions as the ones of [15, 3]. Therefore, we have extended both approaches to the Energy-Transport system in the classical region. Numerical results are provided in the last Section of this paper.

Let us finish with few comments on the two proposed strategies for the coupling. The first one is based on the computation of the boundary layers at the interfaces by performing a diffusive limit of interface conditions for the Boltzmann equation (see [15]). Then, we observed that the continuity of the current is satisfied up to the second order. In the second approach we impose an exact conservation of the current, as in the spirit of [3]. This leads to boundary conditions which are close to the ones derived in the 
first approach. Numerical experiments show that current-voltage characteristics are very close with both approaches. The numerical implementation of the second approach being easier, this strategy could be interesting for much more complex system, such as strongly confined structures.

Acknowledgements : The authors acknowledge partial support from the Galilée Project no 25992ND of the Hubert Curien program : "Modèles numériques du transport collisionnel dans des dispositifs nano-électroniques" and from the AGIR Project, funded by Université Joseph Fourier and Grenoble INP: "Electronic COnfined NanOstructure Modeling".

\section{References}

[1] D. N. Arnold, F. Brezzi, Mixed and nonconforming finite element methods : Implementation, postprocessing and error estimates, RAIRO Modél. Math. Anal. Numér. 19 (1985), 7-32.

[2] C. Bardos, R. Santos, R. Sentis, Diffusion approximation and computation of the critical size, Trans. Am. Math. Soc. 284 (1984), 617-649.

[3] M. Baro, N. Ben Abdallah, P. Degond, A. El Ayyadi, A 1D coupled Schrödinger driftdiffusion model including collisions, J. Comp. Phys 203 (2005), 129-153.

[4] N. Ben Abdallah, A hybrid kinetic-quantum model for stationary electron transport, J. Stat. Phys. 90 (1998), no. 3-4, 627-662.

[5] N. Ben Abdallah, J. M. Cáceres, J. A. Carrillo, F. Vecil, A deterministic solver for a hybrid quantum-classical transport model in nanoMOSFETs, J. Comput. Phys. 228 (2009), no. 17, 6553-6571.

[6] N. Ben Abdallah, P. Degond, On a hierarchy of macroscopic models for semiconductors, J. Math. Phys. 37 (1996), 3306-3333.

[7] N. Ben Abdallah, L. Desvillettes, S. Génieys, On the Convergence of the Boltzmann equation for semiconductors towards the Energy Transport model, J. Stat. Phys. 98 (2000), no. 3-4, $835-870$.

[8] N. Ben Abdallah, C. Jourdana, P. Pietra, An effective mass model for the simulation of ultra-scaled confined devices Math. Models Methods Appl. Sci. 22 (2012), no. 12, 1250039.

[9] N. Ben Abdallah, F. Méhats, Semiclassical analysis of the Schrödinger equation with a partially confining potential, J. Math. Pures App. 84 (2005), 580-614.

[10] N. Ben Abdallah, F. Méhats, C. Negulescu, Adiabatic quantum-fluid transport models, Comm. Math. Sci. 4 (2006), no. 3, 621-650.

[11] N. Ben Abdallah, F. Méhats, N. Vauchelet, Diffusive transport of partially quantized particles : existence uniqueness and long time behaviour, Proc. Edinb. Math. Soc. 49 (2006), 513-549.

[12] N. Ben Abdallah, S. Tang, On hybrid quantum-classical transport models, Math. Meth. Appl. Sci. 27 (2004), 643-667. 
[13] V. D. Camiola, G. Mascali, V. Romano, Simulation of a double-gate MOSFET by a nonparabolic energy-transport model for semiconductors based on the maximum entropy principle, Mathematical and Computer Modelling (2013) 58: 321-343.

[14] D. Chen, E. Kan, U. Ravaioli, C. Shu, and R. Dutton, An improved energy transport model including nonparabolicity and non-Maxwellian distribution effects, IEEE Electr. Device Lett. 13 (1992), 26-28.

[15] P. Degond, A. El Ayyadi, A coupled Schrödinger Drift-Diffusion model for Quantum semiconductor device simulations, J. Comp. Phys. 181 (2002), 222-259.

[16] P. Degond, A. Jüngel, P. Pietra, Numerical discretization of energy-transport models for semiconductors with non-parabolic band structure, SIAM J. Sci. Comput., 22 (2000), 9861007.

[17] P. Degond, J.-G. Liu, L. Mieussens, Macroscopic fluid models with localized kinetic upscaling effects, Multiscale Model. Simul. 5 (2006), no. 3, 940-979.

[18] P. Degond, F. Méhats, C. Ringhofer, Quantum Energy-Transport and Drift-Diffusion models, J. Stat. Phys. 118 (2005), no. 3-4, 625-665.

[19] P. Degond, C. Schmeiser, Macroscopic models for semiconductor heterostructures, J. Math. Phys. 39 (1998), no. 9, 4634-4663.

[20] P. Degond, C. Schmeiser, Kinetic boundary layers and fluid-kinetic coupling in semiconductors, Transp. Theory Statist. Phys. 28 (1999), 31-55.

[21] A. El Ayyadi, A. Jüngel, Semiconductor simulations using a coupled Quantum DriftDiffusion Schrödinger-Poisson model, SIAM J. Appl. Math. 66 (2005), 554-572.

[22] S. Gadau, A. Jüngel, A three-dimensional mixed finite-element approximation of the semiconductor energy-transport equations, SIAM J. Sci. Comput. 31 (2008/09), no 2, 1120-1140.

[23] S. Gadau, A. Jüngel, P. Pietra, A mixed finite-element scheme of a semiconductor energytransport model using dual entropy variables, Hyperbolic problems: theory, numerics and applications. I, 139-146, Yokohama Publ., Yokohama, 2006.

[24] S. Gallego, F. Méhats, Numerical approximation of a quantum drift-diffusion model, C. R. Acad. Sci. Paris, Ser. I 339 (2004), 519-524.

[25] F. Golse, Knudsen layers from a computational viewpoint, Transp. Theory Stat. Phys. 21 (1992), 211-236.

[26] H. K. Gummel, A self-consistent iterative scheme for one-dimensional steady state transistor calculations, IEEE Trans. Electron Devices, 11 (1964), no. 10, 455-465.

[27] C. Jourdana, P. Pietra, A Hybrid Classical-Quantum Transport Model for the Simulation of Carbon Nanotube Transistors, SIAM J. Sci. Comput., 36 (2014), no. 3, B486-B507.

[28] C. Jourdana, N. Vauchelet, Analysis of a diffusive effective mass model for nanowires, Kin. Rel. Models 4 (2011), no. 4, 1121-1142.

[29] A. Jüngel, Transport equations in Semiconductors, Lecture Note in Physics, Springer, 2009. 
[30] A. Jüngel, D. Matthes, A derivation of the isothermal quantum hydrodynamic equations using entropy minimization, ZAMM Z. Angew. Math. Mech. (2005) 85 (11), 806-814.

[31] E. Lyumkis, B. Polsky, A. Shur, and P. Visocky. Transient semiconductor device simulation including energy balance equation, COMPEL 11 (1992), 311-325.

[32] P. A. Markowich, C. A. Ringhofer, C. Schmeiser, Semiconductor equations, Springer-Verlag, Vienna, 1990.

[33] G. Mascali, V. Romano, A non parabolic hydrodynamical subband model for semiconductors based on the maximum entropy principle, Mathematical and Computer Modelling (2012) 55: 10031020 .

[34] P. Pietra, N. Vauchelet, Numerical simulations of an energy-transport model for partially quantized particles, Comm. Math. Sci., 12 (2014), no. 1, 99-123.

[35] O. Pinaud, Transient simulations of a resonant tunneling diode, J. App. Phys. 92 (2002), no. 4, 1987-1994.

[36] R. Stratton, Diffusion of hot and cold electrons in semiconductor barriers, Phys. Rev. 126 (1962), 2002-2014.

[37] R. Stratton, Semiconductor current flow equations (diffusion and degeneracy), IEEE Trans. Electron. Dev. ED-19 (1972), 1288-1292.

[38] S. Q. Tang, D. P. Zhang, Pseudo-Hydrodynamic approximation for transient computaton of energy-transport models in semiconductors, Chinese Physics Letters 22(10) 2633, 2005.

[39] M. L. Tayeb, From Boltzmann equation to spherical harmonics expansion model: diffusion limit and Poisson coupling, Commun. Math. Sci. 9 (2011), no. 1, 255-275.

[40] A. Yamnahaki, Second order boundary conditions for the drift-diffusion equations of semiconductors, Math. Methods Appl. Sci. 5 (1995), 429-456. 